\title{
The zero-level centralizer in endomorphism algebras
}

\author{
Jenő Szigeti and Leon van Wyk
}

\begin{abstract}
For an endomorphism $\varphi \in \operatorname{End}_{R}(M)$ of a left $R$-module ${ }_{R} M$ we investigate the structure and the polynomial identities of the zero-level centralizer $\operatorname{Cen}_{0}(\varphi)$ and the factor $\operatorname{Cen}(\varphi) / \operatorname{Cen}_{0}(\varphi)$. A double zero-centralizer theorem for $\mathrm{Cen}_{0}\left(\mathrm{Cen}_{0}(\varphi)\right)$ is also formulated.
\end{abstract}

\section{INTRODUCTION}

If $S$ is a ring (or algebra), then the centralizer $\operatorname{Cen}(s)=\{u \in S \mid u s=s u\}$ of an element $s \in S$ is a subring (subalgebra) of $S$. We have $\operatorname{Cen}(s)=\bigcup_{c \in \operatorname{LCen}(s)} \operatorname{Cen}_{c}(s)$, where $\operatorname{Cen}_{c}(s)=\{u \in S \mid u s=s u=c\}$ is called the $c$-level centralizer and $\operatorname{LCen}(s)=\left\{c \in S \mid \operatorname{Cen}_{c}(s) \neq \varnothing\right\}$ is a subring of $\operatorname{Cen}(s)$. The zero-level centralizer $\operatorname{Cen}_{0}(s)=\{u \in S \mid u s=s u=0\}$ (or the two-sided annihilator) of $s$ is an ideal of $\operatorname{Cen}(s)$ and $u+\operatorname{Cen}_{0}(s) \longmapsto u s$ is a natural $\operatorname{Cen}(s) / \operatorname{Cen}_{0}(s) \longrightarrow \operatorname{LCen}(s)$ isomorphism of the additive Abelian groups.

The aim of this paper is to investigate the zero-level centralizer $\operatorname{Cen}_{0}(\varphi)$ and the factor $\operatorname{Cen}(\varphi) / \operatorname{Cen}_{0}(\varphi)$ for an element $\varphi$ in the endomorphism ring $\operatorname{End}_{R}(M)$ of a left $R$-module ${ }_{R} M$. Our treatment follows the lines of [DSzW] and is heavily based on the results in $[\mathrm{Sz}]$ and $[\mathrm{DSzW}]$. Thus we restrict our attention to the case of a finitely generated semisimple ${ }_{R} M$. First we focus on a nilpotent $\varphi$ and then we shall see that for a non-nilpotent $\varphi$ the study of $\operatorname{Cen}_{0}(\varphi)$ can be reduced to the nilpotent case.

The authors were not able to find related results in the literature, in spite of the fact that the objects of our investigations arise very naturally. Surprisingly, the dimension formula for the zero-level centralizer of a square matrix has not yet appeared in linear algebra books (e.g. [Ga,P,SuTy,TuA]).

In Section 2 we consider a fixed nilpotent Jordan normal base of ${ }_{R} M$ with respect to a given nilpotent $\varphi \in \operatorname{End}_{R}(M)$ and present all the necessary prerequisites from $[\mathrm{Sz}]$ and $[\mathrm{DSzW}]$.

Section 3 is entirely devoted to the nilpotent case. Theorem 3.3 gives a complete characterization of $\operatorname{Cen}_{0}(\varphi)$ and $\operatorname{Cen}(\varphi) / \operatorname{Cen}_{0}(\varphi)$. If the base ring is local, then a

2010 Mathematics Subject Classification. 15A30, 15A27, 16D60, 16S50, 16 U70.

Key words and phrases. zero-level centralizer of a module endomorphism, nilpotent Jordan normal base.

The authors were supported by the National Research Foundation of South Africa under Grant No. UID 72375. Any opinion, findings and conclusions or recommendations expressed in this material are those of the authors and therefore the National Research Foundation does not accept any liability in regard thereto. 
more accurate description of these algebras can be found in Theorem 3.4. Using 3.4 and the identities of certain subalgebras of a full matrix algebra over $R / J$, in Theorems 3.6 and 3.8 we exhibit explicit polynomial identities for $\operatorname{Cen}_{0}(\varphi)$ and $\operatorname{Cen}(\varphi) / \operatorname{Cen}_{0}(\varphi)$, respectively.

In Section 4 we deal with the non-nilpotent case, a complete description of $\operatorname{Cen}_{0}(\varphi)$ (as a particular ideal of an algebra of certain invariant endomorphisms) can be found in Theorem 4.1. If $A \in M_{n}(K)$ is an $n \times n$ matrix over a field $K$, then the mentioned dimension formula $\operatorname{dim}_{K} \operatorname{Cen}_{0}(A)=\left[\operatorname{dim}_{K}(\operatorname{ker}(A))\right]^{2}$ is an immediate corollary of 4.1. Theorems 4.4 and 4.5 deal with the containment relation $\operatorname{Cen}_{0}(\varphi) \subseteq \operatorname{Cen}_{0}(\sigma)$, where $\sigma \in \operatorname{End}_{R}(M)$ is an other endomorphism. Since this containment is equivalent to $\sigma \in \mathrm{Cen}_{0}\left(\mathrm{Cen}_{0}(\varphi)\right), 4.4$ and 4.5 can be considered as double zero-centralizer theorems.

\section{PREREQUISITES}

In order to provide a self-contained treatment, we collect some notations, definitions and statements from $[\mathrm{Sz}]$ and $[\mathrm{DSzW}]$. Let $Z(R)$ and $J=J(R)$ denote the centre and the Jacobson radical of a ring $R$ (with identity). Let $\left(z^{k}\right) \triangleleft R[z]$ denote the ideal generated by $z^{k}$ in the ring $R[z]$ of polynomials of the commuting indeterminate $z$.

For an $R$-endomorphism $\varphi: M \longrightarrow M$ of a (unitary) left $R$-module ${ }_{R} M$ a subset

$$
\left\{x_{\gamma, i} \mid \gamma \in \Gamma, 1 \leq i \leq k_{\gamma}\right\} \subseteq M
$$

is called a nilpotent Jordan normal base of ${ }_{R} M$ with respect to $\varphi$ if each $R$ submodule $R x_{\gamma, i} \leq M$ is simple, $\underset{\gamma \in \Gamma, 1 \leq i \leq k_{\gamma}}{\oplus} R x_{\gamma, i}=M$ is a direct sum, $\varphi\left(x_{\gamma, i}\right)=$ $x_{\gamma, i+1}, \varphi\left(x_{\gamma, k_{\gamma}}\right)=0$ for all $\gamma \in \Gamma, 1 \leq i \leq k_{\gamma}$, and the set $\left\{k_{\gamma} \mid \gamma \in \Gamma\right\}$ of integers is bounded. Now $\Gamma$ is called the set of (Jordan-) blocks and the size of the block $\gamma \in \Gamma$ is the integer $k_{\gamma} \geq 1$.

2.1.Theorem. Let $\varphi \in \operatorname{End}_{R}(M)$ be an $R$-endomorphism of a left $R$-module ${ }_{R} M$. Then the following are equivalent.

1. ${ }_{R} M$ is a semisimple left $R$-module and $\varphi$ is nilpotent of index $n$.

2. There exists a nilpotent Jordan normal base $X=\left\{x_{\gamma, i} \mid \gamma \in \Gamma, 1 \leq i \leq k_{\gamma}\right\}$ of ${ }_{R} M$ with respect to $\varphi$ such that $n=\max \left\{k_{\gamma} \mid \gamma \in \Gamma\right\}$.

2.2.Theorem. Let $\varphi \in \operatorname{End}_{R}(M)$ be a nilpotent $R$-endomorphism of a finitely generated semisimple left $R$-module ${ }_{R} M$. If

$$
\left\{x_{\gamma, i} \mid \gamma \in \Gamma, 1 \leq i \leq k_{\gamma}\right\} \text { and }\left\{y_{\delta, j} \mid \delta \in \Delta, 1 \leq j \leq l_{\delta}\right\}
$$

are nilpotent Jordan normal bases of ${ }_{R} M$ with respect to $\varphi$, then $\Gamma$ is finite and there exists a bijection $\pi: \Gamma \longrightarrow \Delta$ such that $k_{\gamma}=l_{\pi(\gamma)}$ for all $\gamma \in \Gamma$. Thus the sizes of the blocks of a nilpotent Jordan normal base are unique up to a permutation of the blocks. We also have $\operatorname{ker}(\varphi)=\underset{\gamma \in \Gamma}{\oplus} R x_{\gamma, k_{\gamma}}$ and hence $\operatorname{dim}_{R}(\operatorname{ker}(\varphi))=|\Gamma|$.

If $\varphi \in \operatorname{End}_{R}(M)$ is an arbitrary $R$-endomorphism of the left $R$-module ${ }_{R} M$, then for $u \in M$ and $f(z)=a_{1}+a_{2} z+\cdots+a_{n+1} z^{n} \in R[z]$ (unusual use of indices!) the multiplication

$$
f(z) * u=a_{1} u+a_{2} \varphi(u)+\cdots+a_{n+1} \varphi^{n}(u)
$$


defines a natural left $R[z]$-module structure on $M$. This left action of $R[z]$ on $M$ extends the left action of $R$ on ${ }_{R} M$. For any $R$-endomorphism $\psi \in \operatorname{End}_{R}(M)$ with $\psi \circ \varphi=\varphi \circ \psi$ we have $\psi(f(z) * u)=f(z) * \psi(u)$ and hence $\psi: M \longrightarrow M$ is an $R[z]$ endomorphism of the left $R[z]$-module ${ }_{R[z]} M$. On the other hand, if $\psi: M \longrightarrow M$ is an $R[z]$-endomorphism of ${ }_{R[z]} M$, then $\psi(\varphi(u))=\psi(z * u)=z * \psi(u)=\varphi(\psi(u))$ implies that $\psi \circ \varphi=\varphi \circ \psi$. Now $\operatorname{Cen}(\varphi)=\left\{\psi \mid \psi \in \operatorname{End}_{R}(M)\right.$ and $\left.\psi \circ \varphi=\varphi \circ \psi\right\}$ is a $Z(R)$-subalgebra of $\operatorname{End}_{R}(M)$ and the argument above gives that $\operatorname{Cen}(\varphi)=$ $\operatorname{End}_{R[z]}(M)$.

Henceforth ${ }_{R} M$ is semisimple and we consider a fixed nilpotent Jordan normal base

$$
X=\left\{x_{\gamma, i} \mid \gamma \in \Gamma, 1 \leq i \leq k_{\gamma}\right\} \subseteq M
$$

with respect to a given nilpotent $\varphi \in \operatorname{End}_{R}(M)$ of index $n=\max \left\{k_{\gamma} \mid \gamma \in \Gamma\right\}$.

The $\Gamma$-copower $\amalg_{\gamma \in \Gamma} R[z]$ is an ideal of the $\Gamma$-direct power ring $(R[z])^{\Gamma}$ comprising all elements $\mathbf{f}=\left(f_{\gamma}(z)\right)_{\gamma \in \Gamma}$ with a finite set $\left\{\gamma \in \Gamma \mid f_{\gamma}(z) \neq 0\right\}$ of non-zero coordinates. The copower (power) has a natural $(R[z], R[z])$-bimodule structure. For an element $\mathbf{f}=\left(f_{\gamma}(z)\right)_{\gamma \in \Gamma}$ with $f_{\gamma}(z)=a_{\gamma, 1}+a_{\gamma, 2} z+\cdots+a_{\gamma, n_{\gamma}+1} z^{n_{\gamma}}$ the formula

$$
\Phi(\mathbf{f})=\sum_{\gamma \in \Gamma, 1 \leq i \leq k_{\gamma}} a_{\gamma, i} x_{\gamma, i}=\sum_{\gamma \in \Gamma}\left(\sum_{1 \leq i \leq k_{\gamma}} a_{\gamma, i} \varphi^{i-1}\left(x_{\gamma, 1}\right)\right)=\sum_{\gamma \in \Gamma} f_{\gamma}(z) * x_{\gamma, 1}
$$

defines a function $\Phi: \amalg_{\gamma \in \Gamma} R[z] \rightarrow M$.

2.3.Lemma. The function $\Phi$ is a surjective left $R[z]$-homomorphism. We have $\varphi(\Phi(\mathbf{f}))=\Phi(z \mathbf{f})$ for all $\mathbf{f} \in \amalg_{\gamma \in \Gamma} R[z]$ and the kernel

$$
\coprod_{\gamma \in \Gamma} J[z]+\left(z^{k_{\gamma}}\right) \subseteq \operatorname{ker}(\Phi) \triangleleft_{l} \prod_{\gamma \in \Gamma} R[z]
$$

is a left ideal of the power (and hence of the copower) ring. If $R$ is a local ring $\left(R / J\right.$ is a division ring), then $\amalg_{\gamma \in \Gamma}\left(J[z]+\left(z^{k_{\gamma}}\right)\right)=\operatorname{ker}(\Phi)$.

From now onward we also require that ${ }_{R} M$ be finitely generated, $m=\operatorname{dim}_{R}(\operatorname{ker}(\varphi))$, $\Gamma=\{1,2, \ldots, m\}$ and we assume that $k_{1} \geq k_{2} \geq \ldots \geq k_{m} \geq 1$ for the block sizes. Now $\amalg_{\gamma \in \Gamma} R[z]=(R[z])^{\Gamma}$ and an element $\mathbf{f}=\left(f_{\gamma}(z)\right)_{\gamma \in \Gamma}$ of $(R[z])^{\Gamma}$ is a $1 \times m$ matrix (row vector) over $R[z]$. For an $m \times m$ matrix $\mathbf{P}=\left[p_{\delta, \gamma}(z)\right]$ in $\mathrm{M}_{m}(R[z])$ the matrix product

$$
\mathbf{f P}=\sum_{\delta \in \Gamma} f_{\delta}(z) \mathbf{p}_{\delta}
$$

of $\mathbf{f}$ and $\mathbf{P}$ is a $1 \times m$ matrix over $(R[z])^{\Gamma}$, where $\mathbf{p}_{\delta}=\left(p_{\delta, \gamma}(z)\right)_{\gamma \in \Gamma}$ is the $\delta$-th row vector of $\mathbf{P}$ and

$$
(\mathbf{f P})_{\gamma}=\sum_{\delta \in \Gamma} f_{\delta}(z) p_{\delta, \gamma}(z)
$$

Consider the following subsets of $\mathrm{M}_{m}(R[z])$.

$$
\begin{gathered}
\mathcal{M}(X)=\left\{\mathbf{P} \in \mathrm{M}_{m}(R[z]) \mid \mathbf{f P} \in \operatorname{ker}(\Phi) \text { for all } \mathbf{f} \in \operatorname{ker}(\Phi)\right\}, \\
\mathcal{I}(X)=\left\{\mathbf{P} \in \mathrm{M}_{m}(R[z]) \mid \mathbf{P}=\left[p_{\delta, \gamma}(z)\right] \text { and } p_{\delta, \gamma}(z) \in J[z]+\left(z^{k_{\gamma}}\right) \text { for all } \delta, \gamma \in \Gamma\right\}= \\
=\left[\begin{array}{cccc}
J[z]+\left(z^{k_{1}}\right) & J[z]+\left(z^{k_{2}}\right) & \cdots & J[z]+\left(z^{k_{m}}\right) \\
J[z]+\left(z^{k_{1}}\right) & J[z]+\left(z^{k_{2}}\right) & \cdots & J[z]+\left(z^{k_{m}}\right) \\
\vdots & \vdots & \ddots & \vdots \\
J[z]+\left(z^{k_{1}}\right) & J[z]+\left(z^{k_{2}}\right) & \cdots & J[z]+\left(z^{k_{m}}\right)
\end{array}\right],
\end{gathered}
$$


$\mathcal{N}(X)=\left\{\mathbf{P} \in \mathrm{M}_{m}(R[z]) \mid \mathbf{P}=\left[p_{\delta, \gamma}(z)\right]\right.$ and $z^{k_{\delta}} p_{\delta, \gamma}(z) \in J[z]+\left(z^{k_{\gamma}}\right)$ for all $\left.\delta, \gamma \in \Gamma\right\}$.

Note that $\mathcal{I}(X)$ and $\mathcal{N}(X)$ are $(R[z], R[z])$-sub-bimodules of $\mathrm{M}_{m}(R[z])$ in a natural way. For $\delta, \gamma \in \Gamma$ let $k_{\delta, \gamma}=k_{\gamma}-k_{\delta}$ when $1 \leq k_{\delta}<k_{\gamma} \leq n$ and $k_{\delta, \gamma}=0$ otherwise. It can be verified that the condition $z^{k_{\delta}} p_{\delta, \gamma}(z) \in J[z]+\left(z^{k_{\gamma}}\right)$ in the definition of $\mathcal{N}(X)$ is equivalent to $p_{\delta, \gamma}(z) \in J[z]+\left(z^{k_{\delta, \gamma}}\right)$ and so

$$
\mathcal{N}(X)=\left[\begin{array}{ccccc}
R[z] & R[z] & R[z] & \cdots & R[z] \\
J[z]+\left(z^{k_{1}-k_{2}}\right) & R[z] & R[z] & \cdots & R[z] \\
J[z]+\left(z^{k_{1}-k_{3}}\right) & J[z]+\left(z^{k_{2}-k_{3}}\right) & R[z] & \cdots & R[z] \\
\vdots & \vdots & \vdots & \ddots & \vdots \\
J[z]+\left(z^{k_{1}-k_{m}}\right) & J[z]+\left(z^{k_{2}-k_{m}}\right) & J[z]+\left(z^{k_{3}-k_{m}}\right) & \cdots & R[z]
\end{array}\right]
$$

2.4.Lemma. $\mathcal{I}(X) \triangleleft_{l} \mathrm{M}_{m}(R[z])$ is a left ideal, $\mathcal{N}(X) \subseteq \mathrm{M}_{m}(R[z])$ is a subring, $\mathcal{I}(X) \triangleleft \mathcal{N}(X)$ is an ideal and $\mathcal{M}(X)$ is a $Z(R)$-subalgebra of $\mathrm{M}_{m}(R[z])$. The ideal $z \mathrm{M}_{m}(R[z]) \triangleleft \mathrm{M}_{m}(R[z])$ is nilpotent modulo $\mathcal{I}(X)$ with $\left(z \mathrm{M}_{m}(R[z])\right)^{n} \subseteq \mathcal{I}(X)$. If $R$ is a local ring, then $\mathcal{N}(X)=\mathcal{M}(X)$.

2.5.Theorem. Let $\varphi \in \operatorname{End}_{R}(M)$ be a nilpotent $R$-endomorphism of a finitely generated semisimple left $R$-module ${ }_{R} M$. For $\mathbf{P} \in \mathcal{M}(X)$ and $\mathbf{f}=\left(f_{\gamma}(z)\right)_{\gamma \in \Gamma}$ in $(R[z])^{\Gamma}$ the formula

$$
\psi_{\mathbf{P}}(\Phi(\mathbf{f}))=\Phi(\mathbf{f P})
$$

properly defines an $R$-endomorphism $\psi_{\mathbf{P}}: M \rightarrow M$ of ${ }_{R} M$ such that $\psi_{\mathbf{P}} \circ \varphi=\varphi \circ \psi_{\mathbf{P}}$ and the assignment $\Lambda(\mathbf{P})=\psi_{\mathbf{P}}$ gives an $\mathcal{M}(X)^{\mathrm{op}} \longrightarrow \mathrm{Cen}(\varphi)$ homomorphism of $Z(R)$-algebras. If $\psi \circ \varphi=\varphi \circ \psi$ holds for some $\psi \in \operatorname{End}_{R}(M)$, then there exists an $m \times m$ matrix $\mathbf{P} \in \mathcal{M}(X)$ such that $\psi(\Phi(\mathbf{f}))=\Phi(\mathbf{f P})$ for all $\mathbf{f}=\left(f_{\gamma}(z)\right)_{\gamma \in \Gamma}$ in $(R[z])^{\Gamma}$. Thus $\Lambda: \mathcal{M}(X)^{\mathrm{op}} \longrightarrow \operatorname{Cen}(\varphi)$ is surjective.

2.6.Lemma. $\mathcal{I}(X) \subseteq \operatorname{ker}(\Lambda)$ ( $\Lambda$ is defined in Theorem 2.5). If $R$ is a local ring then $\mathcal{I}(X)=\operatorname{ker}(\Lambda)$.

\section{THE ZERO-LEVEL CENTRALIZER OF A NILPOTENT ENDOMORPHISM}

We keep all settings from Section 2 and define the subsets of $\mathrm{M}_{m}(R[z])$ as follows:

$$
\mathcal{M}_{0}(X)=\left\{\mathbf{P} \in \mathcal{M}(X) \mid z \mathbf{f P} \in \operatorname{ker}(\Phi) \text { for all } \mathbf{f} \in(R[z])^{\Gamma}\right\},
$$

$\mathcal{N}_{0}(X)=\left\{\mathbf{P} \in \mathrm{M}_{m}(R[z]) \mid \mathbf{P}=\left[p_{\delta, \gamma}(z)\right]\right.$ and $p_{\delta, \gamma}(z) \in J[z]+\left(z^{k_{\gamma}-1}\right)$ for all $\left.\delta, \gamma \in \Gamma\right\}$. Since $p_{\delta, \gamma}(z) \in J[z]+\left(z^{k_{\gamma}-1}\right)$ and $z p_{\delta, \gamma}(z) \in J[z]+\left(z^{k_{\gamma}}\right)$ are equivalent, we have

$$
\mathcal{N}_{0}(X)=\left[\begin{array}{cccc}
J[z]+\left(z^{k_{1}-1}\right) & J[z]+\left(z^{k_{2}-1}\right) & \cdots & J[z]+\left(z^{k_{m}-1}\right) \\
J[z]+\left(z^{k_{1}-1}\right) & J[z]+\left(z^{k_{2}-1}\right) & \cdots & J[z]+\left(z^{k_{m}-1}\right) \\
\vdots & \vdots & \ddots & \vdots \\
J[z]+\left(z^{k_{1}-1}\right) & J[z]+\left(z^{k_{2}-1}\right) & \cdots & J[z]+\left(z^{k_{m}-1}\right)
\end{array}\right] .
$$

3.1.Lemma. $\mathcal{I}(X) \subseteq \mathcal{N}_{0}(X),\left(z \mathrm{M}_{m}(R[z])\right)^{n-1} \subseteq \mathcal{N}_{0}(X), \mathcal{N}_{0}(X) \triangleleft_{l} \mathrm{M}_{m}(R[z])$ is a left ideal and $\mathcal{N}_{0}(X) \triangleleft \mathcal{N}(X)$ is an ideal. If $R$ is a local ring, then $\mathcal{N}_{0}(X)=\mathcal{M}_{0}(X)$.

Proof. The containment $\mathcal{I}(X) \subseteq \mathcal{N}_{0}(X)$ obviously holds and $\left(z \mathrm{M}_{m}(R[z])\right)^{n-1} \subseteq$ $\mathcal{N}_{0}(X)$ is a consequence of $\left(z^{n-1}\right) \subseteq\left(z^{k_{\gamma}-1}\right)$. Since the $\gamma$-th column of the matrices 
in $\mathcal{N}_{0}(X)$ comes from a (left) ideal $J[z]+\left(z^{k_{\gamma}-1}\right)$ of $R[z]$, we can see that $\mathcal{N}_{0}(X)$ is a left ideal of $\mathrm{M}_{m}(R[z])$.

If $\mathbf{P} \in \mathcal{N}_{0}(X)$ and $\mathbf{Q} \in \mathcal{N}(X)$, then we have $z p_{\delta, \tau}(z) \in J[z]+\left(z^{k_{\tau}}\right)$ and $q_{\tau, \gamma}(z) \in$ $J[z]+\left(z^{k_{\tau, \gamma}}\right)$. Since $k_{\tau}+k_{\tau, \gamma} \geq k_{\gamma}$, it follows that $z p_{\delta, \tau}(z) q_{\tau, \gamma}(z) \in J[z]+\left(z^{k_{\gamma}}\right)$. Thus $\mathbf{P Q} \in \mathcal{N}_{0}(X)$ and $\mathcal{N}_{0}(X)$ is an ideal of $\mathcal{N}(X)$.

If $R$ is a local ring, then Lemma 2.3 gives that $\operatorname{ker}(\Phi)=\amalg_{\gamma \in \Gamma}\left(J[z]+\left(z^{k_{\gamma}}\right)\right)$. Let $\mathbf{1}_{\delta}$ denote the vector with 1 in its $\delta$-coordinate and zeros in all other places. If $\mathbf{P} \in \mathcal{M}_{0}(X)$, then $z \mathbf{1}_{\delta} \mathbf{P} \in \operatorname{ker}(\Phi)$ implies that $z p_{\delta, \gamma}(z) \in J[z]+\left(z^{k_{\gamma}}\right)$, whence $\mathbf{P} \in \mathcal{N}_{0}(X)$ follows. If $\mathbf{P} \in \mathcal{N}_{0}(X)$ and $\mathbf{f}=\left(f_{\gamma}(z)\right)_{\gamma \in \Gamma}$ is in $(R[z])^{\Gamma}$, then $z p_{\delta, \gamma}(z) \in$ $J[z]+\left(z^{k_{\gamma}}\right)$ implies that $z f_{\delta}(z) p_{\delta, \gamma}(z) \in J[z]+\left(z^{k_{\gamma}}\right)$ for all $\delta \in \Gamma$. Thus $z \mathbf{f P} \in \operatorname{ker}(\Phi)$ and $\mathbf{P} \in \mathcal{M}_{0}(X)$ follows.

3.2.Lemma. $\operatorname{ker}(\Lambda) \subseteq \mathcal{M}_{0}(X)$ and for $\mathbf{P} \in \mathcal{M}(X)$ the containments $\mathbf{P} \in \mathcal{M}_{0}(X)$ and $\Lambda(\mathbf{P}) \in \operatorname{Cen}_{0}(\varphi)$ are equivalent. The preimage $\mathcal{M}_{0}(X)=\Lambda^{-1}\left(\operatorname{Cen}_{0}(\varphi)\right) \triangleleft \mathcal{M}(X)$ is anideal.

Proof. The proof is based on the use of Lemma 2.3 and Theorem 2.5.

If $\mathbf{P} \in \operatorname{ker}(\Lambda)$, then $\Lambda(\mathbf{P})=\psi_{\mathbf{P}}=0$ gives that $\Phi(\mathbf{f P})=\psi_{\mathbf{P}}(\Phi(\mathbf{f}))=0$ for all $\mathbf{f} \in(R[z])^{\Gamma}$. Since $\Phi: \amalg_{\gamma \in \Gamma} R[z] \rightarrow M$ is a left $R[z]$-homomorphism, $\Phi(z \mathbf{f P})=$ $z * \Phi(\mathbf{f P})=0$ implies that $z \mathbf{f P} \in \operatorname{ker}(\Phi)$. In view of $\operatorname{ker}(\Lambda) \subseteq \mathcal{M}(X)$, we deduce that $\mathbf{P} \in \mathcal{M}_{0}(X)$.

If $\mathbf{P} \in \mathcal{M}_{0}(X)$, then $\Lambda(\mathbf{P})=\psi_{\mathbf{P}}$ and $\varphi\left(\psi_{\mathbf{P}}(\Phi(\mathbf{f}))\right)=\varphi(\Phi(\mathbf{f P}))=\Phi(z \mathbf{f P})=0$ for all $\mathbf{f} \in(R[z])^{\Gamma}$. Thus $\psi_{\mathbf{P}} \circ \varphi=\varphi \circ \psi_{\mathbf{P}}=0$ and hence $\psi_{\mathbf{P}} \in \operatorname{Cen}_{0}(\varphi)$.

If $\Lambda(\mathbf{P})=\psi_{\mathbf{P}}$ is in $\operatorname{Cen}_{0}(\varphi)$, then $\varphi \circ \psi_{\mathbf{P}}=0$ and

$$
\Phi(z \mathbf{f P})=\varphi(\Phi(\mathbf{f P}))=\varphi\left(\psi_{\mathbf{P}}(\Phi(\mathbf{f}))\right)=0
$$

for all $\mathbf{f} \in(R[z])^{\Gamma}$. It follows that $\mathbf{P} \in \mathcal{M}_{0}(X)$.

Obviously, the preimage of the ideal $\operatorname{Cen}_{0}(\varphi) \triangleleft \operatorname{Cen}(\varphi)$ is also an ideal.

3.3.Theorem. Let $\varphi \in \operatorname{End}_{R}(M)$ be a nilpotent $R$-endomorphism of a finitely generated semisimple left $R$-module ${ }_{R} M$. The map $\Lambda: \mathcal{M}(X)^{\mathrm{op}} \longrightarrow \operatorname{Cen}(\varphi)$ induces the following $Z(R)$-isomorphisms for the factor algebras:

$$
\mathcal{M}_{0}(X)^{\mathrm{op}} / \operatorname{ker}(\Lambda) \cong \operatorname{Cen}_{0}(\varphi) \text { and } \mathcal{M}(X)^{\mathrm{op}} / \mathcal{M}_{0}(X) \cong \operatorname{Cen}(\varphi) / \operatorname{Cen}_{0}(\varphi) \text {. }
$$

Proof. We have $\operatorname{ker}\left(\Lambda \uparrow \mathcal{M}_{0}(X)\right)=\operatorname{ker}(\Lambda)$ and $\mathcal{M}_{0}(X)=\Lambda^{-1}\left(\operatorname{Cen}_{0}(\varphi)\right)$ by Lemma 3.2. Thus Theorem 2.5 ensures that the restricted map $\Lambda \uparrow \mathcal{M}_{0}(X)$ is a surjective $\mathcal{M}_{0}(X)^{\mathrm{op}} \longrightarrow \mathrm{Cen}_{0}(\varphi)$ homomorphism of $Z(R)$-algebras, whence $\mathcal{M}_{0}(X)^{\mathrm{op}} / \operatorname{ker}(\Lambda) \cong \operatorname{Cen}_{0}(\varphi)$ follows.

In view of Lemma 3.2, the assignment

$$
\mathbf{P}+\mathcal{M}_{0}(X) \longmapsto \Lambda(\mathbf{P})+\operatorname{Cen}_{0}(\varphi)
$$

is well-defined and gives an injective $\mathcal{M}(X)^{\mathrm{op}} / \mathcal{M}_{0}(X) \rightarrow \operatorname{Cen}(\varphi) / \operatorname{Cen}_{0}(\varphi)$ homomorphism of $Z(R)$-algebras. The surjectivity of this homomorphism is a consequence of the surjectivity of $\Lambda$ (see Theorem 2.5).

3.4.Theorem. Let $\varphi \in \operatorname{End}_{R}(M)$ be a nilpotent $R$-endomorphism of a finitely generated semisimple left $R$-module ${ }_{R} M$. If $R$ is a local ring, then the zero-level centralizer $\operatorname{Cen}_{0}(\varphi)$ of $\varphi$ is isomorphic to the opposite of the factor $\mathcal{N}_{0}(X) / \mathcal{I}(X)$ as a $Z(R)$-algebra:

$$
\operatorname{Cen}_{0}(\varphi) \cong\left(\mathcal{N}_{0}(X) / \mathcal{I}(X)\right)^{\mathrm{op}}=\mathcal{N}_{0}(X)^{\mathrm{op}} / \mathcal{I}(X)
$$


We also have an isomorphism

$$
\operatorname{Cen}(\varphi) / \operatorname{Cen}_{0}(\varphi) \cong\left(\mathcal{N}(X) / \mathcal{N}_{0}(X)\right)^{\mathrm{op}}=\mathcal{N}(X)^{\mathrm{op}} / \mathcal{N}_{0}(X)
$$

of the factor $Z(R)$-algebras.

Proof. Directly follows from Lemmas 2.4, 2.6, 3.1 and Theorem 3.3.

Define a left ideal of $\mathrm{M}_{m}(R / J)$ as follows:

$$
\mathcal{W}(X)=\left\{W=\left[w_{\delta, \gamma}\right] \mid w_{\delta, \gamma} \in R / J \text { and } w_{\delta, \gamma}=0 \text { if } k_{\gamma} \geq 2\right\} .
$$

The assumption $k_{1} \geq k_{2} \geq \cdots \geq k_{m} \geq 1$ ensures that

$$
\mathcal{W}(X)=\left[\begin{array}{cccccc}
0 & \cdots & 0 & R / J & \cdots & R / J \\
0 & \ddots & 0 & R / J & \cdots & R / J \\
\vdots & \vdots & \ddots & \vdots & \vdots & \vdots \\
\vdots & \vdots & 0 & R / J & \cdots & R / J \\
\vdots & \vdots & \vdots & \vdots & \ddots & \vdots \\
0 & \cdots & 0 & R / J & \cdots & R / J
\end{array}\right] .
$$

3.5.Lemma. $\left(\mathcal{N}_{0}(X) \cap z \mathrm{M}_{m}(R[z])\right)+\mathcal{I}(X) \triangleleft \mathcal{N}_{0}(X)$ is an ideal and there is a natural ring isomorphism

$$
\mathcal{N}_{0}(X) /\left(\left(\mathcal{N}_{0}(X) \cap z \mathrm{M}_{m}(R[z])\right)+\mathcal{I}(X)\right) \cong \mathcal{W}(X)
$$

which is an $(R, R)$-bimodule isomorphism at the same time.

Proof. If $\mathbf{P}=\left[p_{\delta, \gamma}(z)\right]$ is in $\mathcal{N}_{0}(X)$ and $p_{\delta, \gamma}(z)$ has constant term $u_{\delta, \gamma} \in R$, then

$$
p_{\delta, \gamma}(z)-u_{\delta, \gamma} \in\left(J[z]+\left(z^{k_{\gamma}-1}\right)\right) \cap(z R[z])
$$

and $k_{\gamma} \geq 2$ implies that $u_{\delta, \gamma} \in J$. Thus $\left[u_{\delta, \gamma}\right] \in \mathrm{M}_{m}(R) \cap \mathcal{N}_{0}(X)$ and

$$
\mathbf{P}+\left(\left(\mathcal{N}_{0}(X) \cap z \mathrm{M}_{m}(R[z])\right)+\mathcal{I}(X)\right)=\left[u_{\delta, \gamma}\right]+\left(\left(\mathcal{N}_{0}(X) \cap z \mathrm{M}_{m}(R[z])\right)+\mathcal{I}(X)\right)
$$

holds in $\mathcal{N}_{0}(X) /\left(\left(\mathcal{N}_{0}(X) \cap z \mathrm{M}_{m}(R[z])\right)+\mathcal{I}(X)\right)$. The assignment

$$
\mathbf{P}+\left(\left(\mathcal{N}_{0}(X) \cap z \mathrm{M}_{m}(R[z])\right)+\mathcal{I}(X)\right) \longmapsto\left[u_{\delta, \gamma}+J\right]
$$

is well-defined and gives an

$$
\mathcal{N}_{0}(X) /\left(\left(\mathcal{N}_{0}(X) \cap z \mathrm{M}_{m}(R[z])\right)+\mathcal{I}(X)\right) \longrightarrow \mathcal{W}(X)
$$

isomorphism.

3.6. Theorem. Let $R$ be a local ring and $\varphi \in \operatorname{End}_{R}(M)$ be a nilpotent $R$-endomorphism of a finitely generated semisimple left $R$-module ${ }_{R} M$. If $f_{i}\left(x_{1}, \ldots, x_{r}\right) \in Z(R)\left\langle x_{1}, \ldots, x_{r}\right\rangle$, $1 \leq i \leq n$ and $f_{i}=0$ are polynomial identities of the right ideal $\mathcal{W}(X)$ of $\mathrm{M}_{m}^{\mathrm{op}}(R / J)$, then $f_{1} f_{2} \cdots f_{n}=0$ is an identity of $\operatorname{Cen}_{0}(\varphi)$.

Proof. Theorem 3.4 ensures that $\operatorname{Cen}_{0}(\varphi) \cong \mathcal{N}_{0}(X)^{\text {op }} / \mathcal{I}(X)$ as $Z(R)$-algebras, hence

$$
Q=\left(\left(\mathcal{N}_{0}(X) \cap z \mathrm{M}_{m}(R[z])\right)+\mathcal{I}(X)\right) / \mathcal{I}(X) \triangleleft \mathcal{N}_{0}(X) / \mathcal{I}(X)
$$

can be viewed as an ideal of $\operatorname{Cen}_{0}(\varphi)$. The use of Lemma 3.5 gives $\operatorname{Cen}_{0}(\varphi) / Q \cong\left(\mathcal{N}_{0}(X)^{\mathrm{op}} / \mathcal{I}(X)\right) / Q \cong \mathcal{N}_{0}(X)^{\mathrm{op}} /\left(\mathcal{N}_{0}(X) \cap z \mathrm{M}_{m}(R[z])\right)+\mathcal{I}(X) \cong \mathcal{W}(X)^{\mathrm{op}}$ 
It follows that $f_{i}=0$ is an identity of $\operatorname{Cen}_{0}(\varphi) / Q$. Thus $f_{i}\left(v_{1}, \ldots, v_{r}\right) \in Q$ for all $v_{1}, \ldots, v_{r} \in \operatorname{Cen}_{0}(\varphi)$, and so

$$
f_{1}\left(v_{1}, \ldots, v_{r}\right) f_{2}\left(v_{1}, \ldots, v_{r}\right) \cdots f_{n}\left(v_{1}, \ldots, v_{r}\right) \in Q^{n} .
$$

Since $\left(z \mathrm{M}_{m}(R[z])\right)^{n} \subseteq \mathcal{I}(X)$ (see Lemma 2.4) implies that $Q^{n}=\{0\}$, the proof is complete.

The assumption $k_{1} \geq k_{2} \geq \ldots \geq k_{m} \geq 1$ ensures that

$$
\mathcal{U}_{0}(X)=\left\{U \in \mathrm{M}_{m}(R / J) \mid U=\left[u_{\delta, \gamma}\right] \text { and } u_{\delta, \gamma}=0 \text { if } 1 \leq k_{\delta}<k_{\gamma} \text { or } k_{\gamma}=1\right\} .
$$

is a block upper triangular subalgebra of $\mathrm{M}_{m}(R / J)$. If $\left[u_{\delta, \gamma}\right] \in \mathcal{U}_{0}(X)$ and $u_{\delta, \gamma} \neq 0$ for some $\delta, \gamma \in \Gamma$, then $2 \leq k_{\gamma} \leq k_{\delta}$. Results about the polynomial identities of block upper triangular matrix algebras can be found in [GiZ].

3.7.Lemma. There is a natural ring isomorphism

$$
\mathcal{N}(X) /\left(\left(\mathcal{N}(X) \cap z \mathrm{M}_{m}(R[z])\right)+\mathcal{N}_{0}(X)\right) \cong \mathcal{U}_{0}(X)
$$

which is an $(R, R)$-bimodule isomorphism at the same time.

Proof. For a matrix $\mathbf{P}=\left[p_{\delta, \gamma}(z)\right]$ in $\mathcal{N}(X)$ consider the assignment

$$
\mathbf{P}+\left(\left(\mathcal{N}(X) \cap z \mathrm{M}_{m}(R[z])\right)+\mathcal{N}_{0}(X)\right) \longmapsto\left[u_{\delta, \gamma}+J\right],
$$

where $u_{\delta, \gamma} \in R$ is defined as follows: $u_{\delta, \gamma}=0$ if $k_{\gamma}=1$ and $u_{\delta, \gamma}$ is the constant term of $p_{\delta, \gamma}(z)$ if $k_{\gamma} \geq 2$. Clearly, $\left[u_{\delta, \gamma}\right] \in \mathrm{M}_{m}(R) \cap \mathcal{N}(X)$ and

$\mathbf{P}+\left(\left(\mathcal{N}(X) \cap z \mathrm{M}_{m}(R[z])\right)+\mathcal{N}_{0}(X)\right)=\left[u_{\delta, \gamma}\right]+\left(\left(\mathcal{N}(X) \cap z \mathrm{M}_{m}(R[z])\right)+\mathcal{N}_{0}(X)\right)$.

In view of the definitions of $\mathcal{N}_{0}(X)$ and $\mathcal{U}_{0}(X)$, the above equality ensures that our assignment is a well-defined

$$
\mathcal{N}(X) /\left(\left(\mathcal{N}(X) \cap z \mathrm{M}_{m}(R[z])\right)+\mathcal{N}_{0}(X)\right) \longrightarrow \mathcal{U}_{0}(X)
$$

map providing the required isomomorphism.

3.8. Theorem. Let $R$ be a local ring and $\varphi \in \operatorname{End}_{R}(M)$ be a nilpotent $R$-endomorphism of a finitely generated semisimple left $R$-module ${ }_{R} M$. If $f_{i}\left(x_{1}, \ldots, x_{r}\right) \in Z(R)\left\langle x_{1}, \ldots, x_{r}\right\rangle$, $1 \leq i \leq n-1$ and $f_{i}=0$ are polynomial identities of the $Z(R)$-subalgebra $\mathcal{U}_{0}(X)$ of $\mathrm{M}_{m}^{\mathrm{op}}(R / J)$, then $f_{1} f_{2} \cdots f_{n-1}=0$ is an identity of the factor $\operatorname{Cen}(\varphi) / \operatorname{Cen}_{0}(\varphi)$.

Proof. Theorem 3.4 ensures that $\operatorname{Cen}(\varphi) / \operatorname{Cen}_{0}(\varphi) \cong \mathcal{N}(X)^{\text {op }} / \mathcal{N}_{0}(X)$ as $Z(R)$ algebras, hence

$$
L=\left(\left(\mathcal{N}(X) \cap z \mathrm{M}_{m}(R[z])\right)+\mathcal{N}_{0}(X)\right) / \mathcal{N}_{0}(X) \triangleleft \mathcal{N}(X) / \mathcal{N}_{0}(X)
$$

can be viewed as an ideal of $\operatorname{Cen}(\varphi) / \operatorname{Cen}_{0}(\varphi)$. The use of Lemma 3.7 gives

$$
\begin{gathered}
\left(\operatorname{Cen}(\varphi) / \operatorname{Cen}_{0}(\varphi)\right) / L \cong\left(\mathcal{N}(X)^{\mathrm{op}} / \mathcal{N}_{0}(X)\right) / L \cong \\
\cong \mathcal{N}(X)^{\mathrm{op}} /\left(\left(\mathcal{N}(X) \cap z \mathrm{M}_{m}(R[z])\right)+\mathcal{N}_{0}(X)\right) \cong \mathcal{U}_{0}(X)^{\mathrm{op}} .
\end{gathered}
$$

It follows that $f_{i}=0$ is an identity of $\left(\operatorname{Cen}(\varphi) / \operatorname{Cen}_{0}(\varphi)\right) / L$. Thus $f_{i}\left(v_{1}, \ldots, v_{r}\right) \in$ $L$ for all $v_{1}, \ldots, v_{r} \in \operatorname{Cen}(\varphi) / \operatorname{Cen}_{0}(\varphi)$, and so

$$
f_{1}\left(v_{1}, \ldots, v_{r}\right) f_{2}\left(v_{1}, \ldots, v_{r}\right) \cdots f_{n-1}\left(v_{1}, \ldots, v_{r}\right) \in L^{n-1} .
$$

Since $\left(z \mathrm{M}_{m}(R[z])\right)^{n-1} \subseteq \mathcal{N}_{0}(X)$ (see Lemma 3.1) implies that $L^{n-1}=\{0\}$, the proof is complete. 


\section{THE ZERO-LEVEL CENTRALIZER OF AN ARBITRARY ENDOMORPHISM}

4.1.Theorem. Let $\varphi \in \operatorname{End}_{R}(M)$ be an $R$-endomorphism of a finitely generated semisimple left $R$-module ${ }_{R} M$. Then there exist $R$-submodules $W_{1}, W_{2}$ and $V$ of $M$ such that $W=W_{1} \oplus W_{2}$ and $M=V \oplus W$ are direct products, $\operatorname{ker}(\varphi) \subseteq W$, $\varphi(W)=W_{2}, \varphi(V)=V, \operatorname{dim}_{R}\left(W_{1}\right)=\operatorname{dim}_{R}(\operatorname{ker}(\varphi)),\left(\varphi\lceil W) \in \operatorname{End}_{R}(W)\right.$ is nilpotent and for the zero-level centralizer of $\varphi$ we have $\operatorname{Cen}_{0}(\varphi) \cong T$, where

$$
T=\left\{\theta \in \operatorname{End}_{R}(W) \mid \theta\left(W_{1}\right) \subseteq \operatorname{ker}(\varphi) \text { and } \theta\left(W_{2}\right)=\{0\}\right\}=\operatorname{Cen}_{0}(\varphi\lceil W)
$$

is a left ideal of

$$
\operatorname{End}_{R}^{*}(W)=\left\{\alpha \in \operatorname{End}_{R}(W) \mid \alpha(\operatorname{ker}(\varphi)) \subseteq \operatorname{ker}(\varphi)\right\}
$$

and a right ideal of

$$
\operatorname{End}_{R}^{* *}(W)=\left\{\alpha \in \operatorname{End}_{R}(W) \mid \alpha\left(W_{1}+\operatorname{ker}(\varphi)\right) \subseteq W_{1}+\operatorname{ker}(\varphi) \text { and } \alpha\left(W_{2}\right) \subseteq W_{2}\right\} .
$$

Proof. The Fitting Lemma ensures the existence of an integer $t \geq 1$ such that $\operatorname{im}\left(\varphi^{t}\right) \oplus \operatorname{ker}\left(\varphi^{t}\right)=M$ is a direct sum, where the (left) $R$-submodules

$$
V=\operatorname{im}\left(\varphi^{t}\right)=\operatorname{im}\left(\varphi^{t+1}\right)=\cdots \text { and } W=\operatorname{ker}\left(\varphi^{t}\right)=\operatorname{ker}\left(\varphi^{t+1}\right)=\cdots
$$

of ${ }_{R} M$ are uniquely determined by $\varphi$. Clearly, $\varphi(V)=V$ and $\varphi(W) \subseteq W$ and the restricted map $(\varphi \mid W) \in \operatorname{End}_{R}(W)$ is nilpotent of index $q \geq 1$, where $\operatorname{ker}\left(\varphi^{q-1}\right) \neq$ $\operatorname{ker}\left(\varphi^{q}\right)=W$. Since ${ }_{R} W$ is also finitely generated and semisimple, Theorem 2.1 provides a nilpotent Jordan normal base $X=\left\{x_{\gamma, i} \mid \gamma \in \Gamma, 1 \leq i \leq k_{\gamma}\right\}$ of ${ }_{R} W$ with respect to $\varphi\left\lceil W\right.$ (we have $x_{\gamma, k_{\gamma}+1}=0$ and $q=\max \left\{k_{\gamma} \mid \gamma \in \Gamma\right\}$ ). Now $W_{1} \oplus W_{2}=W$ is a direct sum, where

$$
W_{1}=\underset{\gamma \in \Gamma}{\oplus} R x_{\gamma, 1} \text { and } W_{2}=\underset{\gamma \in \Gamma, 1 \leq i \leq k_{\gamma}}{\bigoplus} R x_{\gamma, i+1} .
$$

Now we have $\operatorname{ker}(\varphi) \subseteq \operatorname{ker}\left(\varphi^{t}\right)=W$ and $\operatorname{ker}(\varphi)=\operatorname{ker}(\varphi \uparrow W)=\underset{\gamma \in \Gamma}{\oplus} R x_{\gamma, k_{\gamma}}$ by Theorem 2.2. It follows that

$$
\operatorname{dim}_{R}\left(W_{1}\right)=|\Gamma|=\operatorname{dim}_{R}(\operatorname{ker}(\varphi)) .
$$

The definition of the nilpotent Jordan normal base ensures that $\varphi(W)=W_{2}$. If $\theta \in T$, then

$$
\theta(\operatorname{ker}(\varphi)) \subseteq \theta\left(W_{1} \oplus W_{2}\right)=\theta\left(W_{1}\right)+\theta\left(W_{2}\right) \subseteq \operatorname{ker}(\varphi)
$$

implies that $T$ is a left ideal of $\operatorname{End}_{R}^{*}(W)$ and a right ideal of $\operatorname{End}_{R}^{* *}(W)$. Clearly, $T=\operatorname{Cen}_{0}(\varphi \uparrow W)$ is a consequence of $\varphi(W)=W_{2}$ and the fact that $\theta(W) \subseteq \operatorname{ker}(\varphi)$ for all $\theta \in T$.

If $\alpha \in \operatorname{Cen}_{0}(\varphi)$, then $\alpha \circ \varphi=0$ implies that $\alpha(V)=\{0\}$ and $\alpha\left(x_{\gamma, i+1}\right)=$ $\alpha\left(\varphi\left(x_{\gamma, i}\right)\right)=0$ for $1 \leq i \leq k_{\gamma}-1$. We also have $\varphi \circ \alpha=0$, whence $\varphi\left(\alpha\left(x_{\gamma, 1}\right)\right)=0$ and $\alpha\left(x_{\gamma, 1}\right) \in \operatorname{ker}(\varphi)$ follow. Thus $\alpha\left(W_{2}\right)=\{0\}, \alpha\left(W_{1}\right) \subseteq \operatorname{ker}(\varphi)$ and the assignment $\alpha \longmapsto \alpha \uparrow W$ obviously defines a $\operatorname{Cen}_{0}(\varphi) \longrightarrow T$ ring homomorphism.

If $\alpha, \beta \in \operatorname{Cen}_{0}(\varphi)$ and $\alpha \uparrow W=\beta\lceil W$, then $\alpha(V)=\beta(V)=\{0\}$ and $V \oplus W=M$ ensure that $\alpha=\beta$ proving the injectivity of the above map.

If $\theta \in T$ and $\pi_{W}: V \oplus W \longrightarrow W$ is the natural projection, then $\theta \circ \pi_{W} \in \operatorname{Cen}_{0}(\varphi)$. Indeed, $\varphi \circ \theta \circ \pi_{W}=0$ is a consequence of $\theta(W) \subseteq \operatorname{ker}(\varphi)$ and $\theta \circ \pi_{W} \circ \varphi=0$ is a consequence of $\varphi(W)=W_{2}$ and $\theta\left(W_{2}\right)=\{0\}$. Hence the surjectivity of our assignment follows from $\theta \circ \pi_{W} \uparrow W=\theta$. 
4.2. Corollary. Let $A \in \mathrm{M}_{n}(K)$ be an $n \times n$ matrix over a field $K$, then the $K$-dimension of the zero-level centralizer of $A$ in $\mathrm{M}_{n}(K)$ is

$$
\operatorname{dim}_{K} \operatorname{Cen}_{0}(A)=\left[\operatorname{dim}_{K}(\operatorname{ker}(A))\right]^{2} .
$$

Proof. Now $A \in \operatorname{End}_{K}\left(K^{n}\right)$ and Theorem 4.1 ensures that $\operatorname{Cen}_{0}(A) \cong T$, where

$$
T=\left\{\theta \in \operatorname{End}_{K}(W) \mid \theta\left(W_{1}\right) \subseteq \operatorname{ker}(A) \text { and } \theta\left(W_{2}\right)=\{0\}\right\} .
$$

Our claim follows from the observation that the elements of $T$ and $\operatorname{Hom}_{K}\left(W_{1}, \operatorname{ker}(A)\right)$ can be naturally identified and $\operatorname{dim}_{K}\left(W_{1}\right)=\operatorname{dim}_{K}(\operatorname{ker}(A))$.

Remark. Theorem 4.1 shows that the determination of the zero-level centralizer can be reduced to the nilpotent case. This reduction depends on the use of the Fitting Lemma.

4.3.Lemma. Let $\varphi, \sigma \in \operatorname{End}_{R}(M)$ be R-endomorphisms of a finitely generated semisimple left $R$-module ${ }_{R} M$. If $\operatorname{Cen}_{0}(\varphi) \subseteq \operatorname{Cen}_{0}(\sigma)$, then $\operatorname{ker}(\varphi) \subseteq \operatorname{ker}(\sigma)$ and $\operatorname{im}(\sigma) \subseteq \operatorname{im}(\varphi)$.

Proof. We use the proof of Theorem 4.1. If $\gamma \in \Gamma$ and $\pi_{\gamma} \in \operatorname{End}_{R}(M)$ denotes the natural

$$
M=V \oplus W=V \oplus\left(\underset{\delta \in \Gamma, 1 \leq i \leq k_{\delta}}{\bigoplus_{\delta, i}} R x\right) \rightarrow R x_{\gamma, k_{\gamma}}
$$

projection, then $\pi_{\gamma} \circ \varphi^{k_{\gamma}-1} \in \operatorname{Cen}_{0}(\varphi)$. It follows that $\pi_{\gamma} \circ \varphi^{k_{\gamma}-1} \in \operatorname{Cen}_{0}(\sigma)$, whence we obtain that $\pi_{\gamma} \circ \varphi^{k_{\gamma}-1} \circ \sigma=\sigma \circ \pi_{\gamma} \circ \varphi^{k_{\gamma}-1}=0$. Since $\sigma\left(x_{\gamma, k_{\gamma}}\right)=$ $\sigma\left(\pi_{\gamma}\left(\varphi^{k_{\gamma}-1}\left(x_{\gamma, k_{1}}\right)\right)\right)=0$, we have $x_{\gamma, k_{\gamma}} \in \operatorname{ker}(\sigma)$ for all $\gamma \in \Gamma$. Thus

$$
\operatorname{ker}(\varphi)=\operatorname{ker}(\varphi \mid W)=\underset{\gamma \in \Gamma}{\oplus} R x_{\gamma, k_{\gamma}} \subseteq \operatorname{ker}(\sigma) .
$$

The containment $\operatorname{im}(\sigma) \subseteq \operatorname{ker}\left(\pi_{\gamma} \circ \varphi^{k_{\gamma}-1}\right)$ is a consequence of $\pi_{\gamma} \circ \varphi^{k_{\gamma}-1} \circ \sigma=0$, whence we obtain that $\operatorname{im}(\sigma) \subseteq \cap_{\gamma \in \Gamma} \operatorname{ker}\left(\pi_{\gamma} \circ \varphi^{k_{\gamma}-1}\right)$. It is straightforward to see that $\operatorname{ker}\left(\pi_{\gamma} \circ \varphi^{k_{\gamma}-1}\right)=V \oplus W(\gamma)$ and

$$
\bigcap_{\gamma \in \Gamma}(V \oplus W(\gamma))=V \oplus W_{2}=\varphi(V)+\varphi(W)=\varphi(V \oplus W)=\operatorname{im}(\varphi),
$$

where

$$
W(\gamma)=\bigoplus_{\delta \in \Gamma, 1 \leq i \leq k_{\delta},(\delta, i) \neq(\gamma, 1)} R x_{\delta, i} .
$$

4.4.Theorem. Let $\varphi, \sigma \in \operatorname{End}_{R}(M)$ be R-endomorphisms of a finitely generated semisimple left $R$-module ${ }_{R} M$, then the following are equivalent:

1. $\operatorname{Cen}_{0}(\varphi) \subseteq \operatorname{Cen}_{0}(\sigma)$,

2. $\operatorname{ker}(\varphi) \subseteq \operatorname{ker}(\sigma)$ and $\operatorname{im}(\sigma) \subseteq \operatorname{im}(\varphi)$.

Proof. In view of Lemma 4.3, it is enough to prove $(2) \Longrightarrow(1)$. For an endomorphism $\tau \in \operatorname{Cen}_{0}(\varphi)$ we have $\tau \circ \varphi=\varphi \circ \tau=0$, whence $\operatorname{im}(\sigma) \subseteq \operatorname{im}(\varphi) \subseteq \operatorname{ker}(\tau)$ and $\operatorname{im}(\tau) \subseteq \operatorname{ker}(\varphi) \subseteq \operatorname{ker}(\sigma)$ follow. Thus we obtain that $\tau \circ \sigma=\sigma \circ \tau=0$. In consequence we have $\tau \in \operatorname{Cen}_{0}(\sigma)$ and $\operatorname{Cen}_{0}(\varphi) \subseteq \operatorname{Cen}_{0}(\sigma)$ follows.

For a matrix $A \in \mathrm{M}_{n}(K)$ let $A^{\top}$ denote the transpose of $A$. 
4.5.Theorem. If $A, B \in \mathrm{M}_{n}(K)$ are $n \times n$ matrices over a field $K$, then the following are equivalent:

1. $\operatorname{Cen}_{0}(A) \subseteq \operatorname{Cen}_{0}(B)$,

2. $\operatorname{ker}(A) \subseteq \operatorname{ker}(B)$ and $\operatorname{ker}\left(A^{\top}\right) \subseteq \operatorname{ker}\left(B^{\top}\right)$,

3. $\operatorname{im}(B) \subseteq \operatorname{im}(A)$ and $\operatorname{im}\left(B^{\top}\right) \subseteq \operatorname{im}\left(A^{\top}\right)$.

Proof. (1) $\Longrightarrow(2) \&(3)$ : For a matrix $C \in \operatorname{Cen}_{0}\left(A^{\top}\right)$ we have $C A^{\top}=A^{\top} C=0$ and $C^{\top} \in \operatorname{Cen}_{0}(A)$ is a consequence of

$$
A C^{\top}=\left(A^{\top}\right)^{\top} C^{\top}=\left(C A^{\top}\right)^{\top}=0=\left(A^{\top} C\right)^{\top}=C^{\top}\left(A^{\top}\right)^{\top}=C^{\top} A .
$$

Thus $C^{\top} \in \operatorname{Cen}_{0}(B)$ and a similar argument gives that $C=\left(C^{\top}\right)^{\top} \in \operatorname{Cen}_{0}\left(B^{\top}\right)$. It follows that $\operatorname{Cen}_{0}\left(A^{\top}\right) \subseteq \operatorname{Cen}_{0}\left(B^{\top}\right)$. The application of Lemma 4.3 for the matrices $A, B, A^{\top}, B^{\top} \in \operatorname{End}_{K}\left(K^{n}\right)$ gives $\operatorname{ker}(A) \subseteq \operatorname{ker}(B), \operatorname{im}(B) \subseteq \operatorname{im}(A)$ and $\operatorname{ker}\left(A^{\top}\right) \subseteq \operatorname{ker}\left(B^{\top}\right), \operatorname{im}\left(B^{\top}\right) \subseteq \operatorname{im}\left(A^{\top}\right)$.

$(2) \Longrightarrow(1)$ : For a matrix $C \in \operatorname{Cen}_{0}(A)$ the containment $\operatorname{im}(C) \subseteq \operatorname{ker}(A)$ is a consequence of $A C=0$ and $\operatorname{im}\left(C^{\top}\right) \subseteq \operatorname{ker}\left(A^{\top}\right)$ is a consequence of $A^{\top} C^{\top}=$ $(C A)^{\top}=0$. Now $\operatorname{im}(C) \subseteq \operatorname{ker}(B)$ implies that $B C=0$ and $\operatorname{im}\left(C^{\top}\right) \subseteq \operatorname{ker}\left(B^{\top}\right)$ implies that $C B=\left(B^{\top} C^{\top}\right)^{\top}=0$. Thus $C \in \operatorname{Cen}_{0}(B)$ and $\operatorname{Cen}_{0}(A) \subseteq \operatorname{Cen}_{0}(B)$ follows.

$(3) \Longrightarrow(1)$ : For a matrix $C \in \operatorname{Cen}_{0}(A)$ the containment $\operatorname{im}(A) \subseteq \operatorname{ker}(C)$ is a consequence of $C A=0$ and $\operatorname{im}\left(A^{\top}\right) \subseteq \operatorname{ker}\left(C^{\top}\right)$ is a consequence of $C^{\top} A^{\top}=$ $(A C)^{\top}=0$. Now $\operatorname{im}(B) \subseteq \operatorname{ker}(C)$ implies that $C B=0$ and $\operatorname{im}\left(B^{\top}\right) \subseteq \operatorname{ker}\left(C^{\top}\right)$ implies that $B C=\left(C^{\top} B^{\top}\right)^{\top}=0$. Thus $C \in \operatorname{Cen}_{0}(B)$ and $\operatorname{Cen}_{0}(A) \subseteq \operatorname{Cen}_{0}(B)$ follows.

ACKNOWLEDGMENT: The authors wish to thank P.N. Anh and L. Marki for fruitful consultations.

\section{REFERENCES}

[DSzW] Drensky, V., Szigeti, J. and van Wyk, L. Centralizers in endomorphism rings, J. Algebra 324 (2010), 3378-3387.

[Ga] Gantmacher, F.R. The Theory of Matrices, Chelsea Publishing Co., New York, 2000.

[GiZ] Giambruno, A. and Zaicev, M. Polynomial Identities and Asymptotic Methods, Mathematical Surveys and Monographs 122, Amer. Math. Soc., Providence, Rhode Island, 2005.

[P] Prasolov, V.V. Problems and Theorems in Linear Algebra, Vol. 134 of Translation of Mathematical Monographs, Amer. Math. Soc., Providence, Rhode Island, 1994.

[SuTy] Suprunenko, D.A. and Tyshkevich, R.I. Commutative Matrices, Academic Press, New York and London, 1968.

[Sz] Szigeti, J. Linear algebra in lattices and nilpotent endomorphisms of semisimple modules, J. Algebra 319 (2008), 296-308. 
[TuA] Turnbull, H.W. and Aitken, A.C. An Introduction to the Theory of Canonical Matrices, Dover Publications, 2004.

Institute of Mathematics, University of Miskolc, Miskolc, Hungary 3515

E-mail address: jeno.szigeti@uni-miskolc.hu

Department of Mathematical Sciences, Stellenbosch University, P/Bag X1, Matieland 7602, Stellenbosch, South Africa

E-mail address: LvW@sun.ac.za 


\title{
The zero-level centralizer in endomorphism algebras
}

\author{
Jenő Szigeti and Leon van Wyk
}

\begin{abstract}
For an endomorphism $\varphi \in \operatorname{End}_{R}(M)$ of a left $R$-module ${ }_{R} M$ we investigate the structure and the polynomial identities of the zero-level centralizer $\operatorname{Cen}_{0}(\varphi)$ and the factor $\operatorname{Cen}(\varphi) / \operatorname{Cen}_{0}(\varphi)$. A double zero-centralizer theorem for $\mathrm{Cen}_{0}\left(\mathrm{Cen}_{0}(\varphi)\right)$ is also formulated.
\end{abstract}

\section{INTRODUCTION}

If $S$ is a ring (or algebra), then the centralizer $\operatorname{Cen}(s)=\{u \in S \mid u s=s u\}$ of an element $s \in S$ is a subring (subalgebra) of $S$. We have $\operatorname{Cen}(s)=\bigcup_{c \in \operatorname{LCen}(s)} \operatorname{Cen}_{c}(s)$, where $\operatorname{Cen}_{c}(s)=\{u \in S \mid u s=s u=c\}$ is called the $c$-level centralizer and $\operatorname{LCen}(s)=\left\{c \in S \mid \operatorname{Cen}_{c}(s) \neq \varnothing\right\}$ is a subring of $\operatorname{Cen}(s)$. The zero-level centralizer $\operatorname{Cen}_{0}(s)=\{u \in S \mid u s=s u=0\}$ (or the two-sided annihilator) of $s$ is an ideal of $\operatorname{Cen}(s)$ and $u+\operatorname{Cen}_{0}(s) \longmapsto u s$ is a natural $\operatorname{Cen}(s) / \operatorname{Cen}_{0}(s) \longrightarrow \operatorname{LCen}(s)$ isomorphism of the additive Abelian groups.

The aim of this paper is to investigate the zero-level centralizer $\operatorname{Cen}_{0}(\varphi)$ and the factor $\operatorname{Cen}(\varphi) / \operatorname{Cen}_{0}(\varphi)$ for an element $\varphi$ in the endomorphism ring $\operatorname{End}_{R}(M)$ of a left $R$-module ${ }_{R} M$. Our treatment follows the lines of [DSzW] and is heavily based on the results in $[\mathrm{Sz}]$ and $[\mathrm{DSzW}]$. Thus we restrict our attention to the case of a finitely generated semisimple ${ }_{R} M$. First we focus on a nilpotent $\varphi$ and then we shall see that for a non-nilpotent $\varphi$ the study of $\operatorname{Cen}_{0}(\varphi)$ can be reduced to the nilpotent case.

The authors were not able to find related results in the literature, in spite of the fact that the objects of our investigations arise very naturally. Surprisingly, the dimension formula for the zero-level centralizer of a square matrix has not yet appeared in linear algebra books (e.g. [Ga,P,SuTy,TuA]).

In Section 2 we consider a fixed nilpotent Jordan normal base of ${ }_{R} M$ with respect to a given nilpotent $\varphi \in \operatorname{End}_{R}(M)$ and present all the necessary prerequisites from $[\mathrm{Sz}]$ and $[\mathrm{DSzW}]$.

Section 3 is entirely devoted to the nilpotent case. Theorem 3.3 gives a complete characterization of $\operatorname{Cen}_{0}(\varphi)$ and $\operatorname{Cen}(\varphi) / \operatorname{Cen}_{0}(\varphi)$. If the base ring is local, then a

2010 Mathematics Subject Classification. 15A30, 15A27, 16D60, 16S50, 16 U70.

Key words and phrases. zero-level centralizer of a module endomorphism, nilpotent Jordan normal base.

The authors were supported by the National Research Foundation of South Africa under Grant No. UID 72375. Any opinion, findings and conclusions or recommendations expressed in this material are those of the authors and therefore the National Research Foundation does not accept any liability in regard thereto. 
more accurate description of these algebras can be found in Theorem 3.4. Using 3.4 and the identities of certain subalgebras of a full matrix algebra over $R / J$, in Theorems 3.6 and 3.8 we exhibit explicit polynomial identities for $\operatorname{Cen}_{0}(\varphi)$ and $\operatorname{Cen}(\varphi) / \operatorname{Cen}_{0}(\varphi)$, respectively.

In Section 4 we deal with the non-nilpotent case, a complete description of $\mathrm{Cen}_{0}(\varphi)$ (as a particular ideal of an algebra of certain invariant endomorphisms) can be found in Theorem 4.1. If $A, B \in M_{n}(K)$ are $n \times n$ matrices over a field $K$, then the mentioned dimension formula is $\operatorname{dim}_{K} \operatorname{Cen}_{0}(A)=\left[\operatorname{dim}_{K}(\operatorname{ker}(A))\right]^{2}$ and Theorem 4.4 deals with the containment relation $\operatorname{Cen}_{0}(A) \subseteq \operatorname{Cen}_{0}(B)$. Since this containment is equivalent to $B \in \mathrm{Cen}_{0}\left(\operatorname{Cen}_{0}(A)\right)$, our result can be considered as a double zero-centralizer theorem.

\section{PREREQUISITES}

In order to provide a self-contained treatment, we collect some notations, definitions and statements from $[\mathrm{Sz}]$ and $[\mathrm{DSzW}]$. Let $Z(R)$ and $J=J(R)$ denote the centre and the Jacobson radical of a ring $R$ (with identity). Let $\left(z^{k}\right) \triangleleft R[z]$ denote the ideal generated by $z^{k}$ in the ring $R[z]$ of polynomials of the commuting indeterminate $z$.

For an $R$-endomorphism $\varphi: M \longrightarrow M$ of a (unitary) left $R$-module ${ }_{R} M$ a subset

$$
\left\{x_{\gamma, i} \mid \gamma \in \Gamma, 1 \leq i \leq k_{\gamma}\right\} \subseteq M
$$

is called a nilpotent Jordan normal base of ${ }_{R} M$ with respect to $\varphi$ if each $R$ submodule $R x_{\gamma, i} \leq M$ is simple, $\underset{\gamma \in \Gamma, 1 \leq i \leq k_{\gamma}}{\oplus} R x_{\gamma, i}=M$ is a direct sum, $\varphi\left(x_{\gamma, i}\right)=$ $x_{\gamma, i+1}, \varphi\left(x_{\gamma, k_{\gamma}}\right)=0$ for all $\gamma \in \Gamma, 1 \leq i \leq k_{\gamma}$, and the set $\left\{k_{\gamma} \mid \gamma \in \Gamma\right\}$ of integers is bounded. Now $\Gamma$ is called the set of (Jordan-) blocks and the size of the block $\gamma \in \Gamma$ is the integer $k_{\gamma} \geq 1$.

2.1.Theorem. Let $\varphi \in \operatorname{End}_{R}(M)$ be an $R$-endomorphism of a left $R$-module ${ }_{R} M$. Then the following are equivalent.

1. ${ }_{R} M$ is a semisimple left $R$-module and $\varphi$ is nilpotent of index $n$.

2. There exists a nilpotent Jordan normal base $X=\left\{x_{\gamma, i} \mid \gamma \in \Gamma, 1 \leq i \leq k_{\gamma}\right\}$ of ${ }_{R} M$ with respect to $\varphi$ such that $n=\max \left\{k_{\gamma} \mid \gamma \in \Gamma\right\}$.

2.2.Theorem. Let $\varphi \in \operatorname{End}_{R}(M)$ be a nilpotent $R$-endomorphism of a finitely generated semisimple left $R$-module ${ }_{R} M$. If

$$
\left\{x_{\gamma, i} \mid \gamma \in \Gamma, 1 \leq i \leq k_{\gamma}\right\} \text { and }\left\{y_{\delta, j} \mid \delta \in \Delta, 1 \leq j \leq l_{\delta}\right\}
$$

are nilpotent Jordan normal bases of ${ }_{R} M$ with respect to $\varphi$, then $\Gamma$ is finite and there exists a bijection $\pi: \Gamma \longrightarrow \Delta$ such that $k_{\gamma}=l_{\pi(\gamma)}$ for all $\gamma \in \Gamma$. Thus the sizes of the blocks of a nilpotent Jordan normal base are unique up to a permutation of the blocks. We also have $\operatorname{ker}(\varphi)=\underset{\gamma \in \Gamma}{\oplus} R x_{\gamma, k_{\gamma}}$ and hence $\operatorname{dim}_{R}(\operatorname{ker}(\varphi))=|\Gamma|$.

If $\varphi \in \operatorname{End}_{R}(M)$ is an arbitrary $R$-endomorphism of the left $R$-module ${ }_{R} M$, then for $u \in M$ and $f(z)=a_{1}+a_{2} z+\cdots+a_{n+1} z^{n} \in R[z]$ (unusual use of indices!) the multiplication

$$
f(z) * u=a_{1} u+a_{2} \varphi(u)+\cdots+a_{n+1} \varphi^{n}(u)
$$

defines a natural left $R[z]$-module structure on $M$. This left action of $R[z]$ on $M$ extends the left action of $R$ on ${ }_{R} M$. For any $R$-endomorphism $\psi \in \operatorname{End}_{R}(M)$ with 
$\psi \circ \varphi=\varphi \circ \psi$ we have $\psi(f(z) * u)=f(z) * \psi(u)$ and hence $\psi: M \longrightarrow M$ is an $R[z]$ endomorphism of the left $R[z]$-module ${ }_{R[z]} M$. On the other hand, if $\psi: M \longrightarrow M$ is an $R[z]$-endomorphism of ${ }_{R[z]} M$, then $\psi(\varphi(u))=\psi(z * u)=z * \psi(u)=\varphi(\psi(u))$ implies that $\psi \circ \varphi=\varphi \circ \psi$. Now $\operatorname{Cen}(\varphi)=\left\{\psi \mid \psi \in \operatorname{End}_{R}(M)\right.$ and $\left.\psi \circ \varphi=\varphi \circ \psi\right\}$ is a $Z(R)$-subalgebra of $\operatorname{End}_{R}(M)$ and the argument above gives that $\operatorname{Cen}(\varphi)=$ $\operatorname{End}_{R[z]}(M)$.

Henceforth ${ }_{R} M$ is semisimple and we consider a fixed nilpotent Jordan normal base

$$
X=\left\{x_{\gamma, i} \mid \gamma \in \Gamma, 1 \leq i \leq k_{\gamma}\right\} \subseteq M
$$

with respect to a given nilpotent $\varphi \in \operatorname{End}_{R}(M)$ of index $n=\max \left\{k_{\gamma} \mid \gamma \in \Gamma\right\}$.

The $\Gamma$-copower $\amalg_{\gamma \in \Gamma} R[z]$ is an ideal of the $\Gamma$-direct power ring $(R[z])^{\Gamma}$ comprising all elements $\mathbf{f}=\left(f_{\gamma}(z)\right)_{\gamma \in \Gamma}$ with a finite set $\left\{\gamma \in \Gamma \mid f_{\gamma}(z) \neq 0\right\}$ of non-zero coordinates. The copower (power) has a natural $(R[z], R[z])$-bimodule structure. For an element $\mathbf{f}=\left(f_{\gamma}(z)\right)_{\gamma \in \Gamma}$ with $f_{\gamma}(z)=a_{\gamma, 1}+a_{\gamma, 2} z+\cdots+a_{\gamma, n_{\gamma}+1} z^{n_{\gamma}}$ the formula

$$
\Phi(\mathbf{f})=\sum_{\gamma \in \Gamma, 1 \leq i \leq k_{\gamma}} a_{\gamma, i} x_{\gamma, i}=\sum_{\gamma \in \Gamma}\left(\sum_{1 \leq i \leq k_{\gamma}} a_{\gamma, i} \varphi^{i-1}\left(x_{\gamma, 1}\right)\right)=\sum_{\gamma \in \Gamma} f_{\gamma}(z) * x_{\gamma, 1}
$$

defines a function $\Phi: \amalg_{\gamma \in \Gamma} R[z] \rightarrow M$.

2.3.Lemma. The function $\Phi$ is a surjective left $R[z]$-homomorphism. We have $\varphi(\Phi(\mathbf{f}))=\Phi(z \mathbf{f})$ for all $\mathbf{f} \in \amalg_{\gamma \in \Gamma} R[z]$ and the kernel

$$
\coprod_{\gamma \in \Gamma} J[z]+\left(z^{k_{\gamma}}\right) \subseteq \operatorname{ker}(\Phi) \triangleleft_{l} \prod_{\gamma \in \Gamma} R[z]
$$

is a left ideal of the power (and hence of the copower) ring. If $R$ is a local ring ( $R / J$ is a division ring), then $\amalg_{\gamma \in \Gamma}\left(J[z]+\left(z^{k_{\gamma}}\right)\right)=\operatorname{ker}(\Phi)$.

From now onward we also require that ${ }_{R} M$ be finitely generated, $m=\operatorname{dim}_{R}(\operatorname{ker}(\varphi))$, $\Gamma=\{1,2, \ldots, m\}$ and we assume that $k_{1} \geq k_{2} \geq \ldots \geq k_{m} \geq 1$ for the block sizes. Now $\amalg_{\gamma \in \Gamma} R[z]=(R[z])^{\Gamma}$ and an element $\mathbf{f}=\left(f_{\gamma}(z)\right)_{\gamma \in \Gamma}$ of $(R[z])^{\Gamma}$ is a $1 \times m$ matrix (row vector) over $R[z]$. For an $m \times m$ matrix $\mathbf{P}=\left[p_{\delta, \gamma}(z)\right]$ in $\mathrm{M}_{m}(R[z])$ the matrix product

$$
\mathbf{f P}=\sum_{\delta \in \Gamma} f_{\delta}(z) \mathbf{p}_{\delta}
$$

of $\mathbf{f}$ and $\mathbf{P}$ is a $1 \times m$ matrix over $(R[z])^{\Gamma}$, where $\mathbf{p}_{\delta}=\left(p_{\delta, \gamma}(z)\right)_{\gamma \in \Gamma}$ is the $\delta$-th row vector of $\mathbf{P}$ and

$$
(\mathbf{f P})_{\gamma}=\sum_{\delta \in \Gamma} f_{\delta}(z) p_{\delta, \gamma}(z)
$$

Consider the following subsets of $\mathrm{M}_{m}(R[z])$.

$$
\mathcal{M}(X)=\left\{\mathbf{P} \in \mathrm{M}_{m}(R[z]) \mid \mathbf{f P} \in \operatorname{ker}(\Phi) \text { for all } \mathbf{f} \in \operatorname{ker}(\Phi)\right\}
$$

$\mathcal{I}(X)=\left\{\mathbf{P} \in \mathrm{M}_{m}(R[z]) \mid \mathbf{P}=\left[p_{\delta, \gamma}(z)\right]\right.$ and $p_{\delta, \gamma}(z) \in J[z]+\left(z^{k_{\gamma}}\right)$ for all $\left.\delta, \gamma \in \Gamma\right\}=$

$$
=\left[\begin{array}{cccc}
J[z]+\left(z^{k_{1}}\right) & J[z]+\left(z^{k_{2}}\right) & \cdots & J[z]+\left(z^{k_{m}}\right) \\
J[z]+\left(z^{k_{1}}\right) & J[z]+\left(z^{k_{2}}\right) & \cdots & J[z]+\left(z^{k_{m}}\right) \\
\vdots & \vdots & \ddots & \vdots \\
J[z]+\left(z^{k_{1}}\right) & J[z]+\left(z^{k_{2}}\right) & \cdots & J[z]+\left(z^{k_{m}}\right)
\end{array}\right],
$$

$\mathcal{N}(X)=\left\{\mathbf{P} \in \mathrm{M}_{m}(R[z]) \mid \mathbf{P}=\left[p_{\delta, \gamma}(z)\right]\right.$ and $z^{k_{\delta}} p_{\delta, \gamma}(z) \in J[z]+\left(z^{k_{\gamma}}\right)$ for all $\left.\delta, \gamma \in \Gamma\right\}$. 
Note that $\mathcal{I}(X)$ and $\mathcal{N}(X)$ are $(R[z], R[z])$-sub-bimodules of $\mathrm{M}_{m}(R[z])$ in a natural way. For $\delta, \gamma \in \Gamma$ let $k_{\delta, \gamma}=k_{\gamma}-k_{\delta}$ when $1 \leq k_{\delta}<k_{\gamma} \leq n$ and $k_{\delta, \gamma}=0$ otherwise. It can be verified that the condition $z^{k_{\delta}} p_{\delta, \gamma}(z) \in J[z]+\left(z^{k_{\gamma}}\right)$ in the definition of $\mathcal{N}(X)$ is equivalent to $p_{\delta, \gamma}(z) \in J[z]+\left(z^{k, \gamma}\right)$ and so

$$
\mathcal{N}(X)=\left[\begin{array}{ccccc}
R[z] & R[z] & R[z] & \cdots & R[z] \\
J[z]+\left(z^{k_{1}-k_{2}}\right) & R[z] & R[z] & \cdots & R[z] \\
J[z]+\left(z^{k_{1}-k_{3}}\right) & J[z]+\left(z^{k_{2}-k_{3}}\right) & R[z] & \cdots & R[z] \\
\vdots & \vdots & \vdots & \ddots & \vdots \\
J[z]+\left(z^{k_{1}-k_{m}}\right) & J[z]+\left(z^{k_{2}-k_{m}}\right) & J[z]+\left(z^{k_{3}-k_{m}}\right) & \cdots & R[z]
\end{array}\right] .
$$

2.4.Lemma. $\mathcal{I}(X) \triangleleft_{l} \mathrm{M}_{m}(R[z])$ is a left ideal, $\mathcal{N}(X) \subseteq \mathrm{M}_{m}(R[z])$ is a subring, $\mathcal{I}(X) \triangleleft \mathcal{N}(X)$ is an ideal and $\mathcal{M}(X)$ is a $Z(R)$-subalgebra of $\mathrm{M}_{m}(R[z])$. The ideal $z \mathrm{M}_{m}(R[z]) \triangleleft \mathrm{M}_{m}(R[z])$ is nilpotent modulo $\mathcal{I}(X)$ with $\left(z \mathrm{M}_{m}(R[z])\right)^{n} \subseteq \mathcal{I}(X)$. If $R$ is a local ring, then $\mathcal{N}(X)=\mathcal{M}(X)$.

2.5.Theorem. Let $\varphi \in \operatorname{End}_{R}(M)$ be a nilpotent $R$-endomorphism of a finitely generated semisimple left $R$-module ${ }_{R} M$. For $\mathbf{P} \in \mathcal{M}(X)$ and $\mathbf{f}=\left(f_{\gamma}(z)\right)_{\gamma \in \Gamma}$ in $(R[z])^{\Gamma}$ the formula

$$
\psi_{\mathbf{P}}(\Phi(\mathbf{f}))=\Phi(\mathbf{f P})
$$

properly defines an $R$-endomorphism $\psi_{\mathbf{P}}: M \rightarrow M$ of ${ }_{R} M$ such that $\psi_{\mathbf{P}} \circ \varphi=\varphi \circ \psi_{\mathbf{P}}$ and the assignment $\Lambda(\mathbf{P})=\psi_{\mathbf{P}}$ gives an $\mathcal{M}(X)^{\mathrm{op}} \longrightarrow \operatorname{Cen}(\varphi)$ homomorphism of $Z(R)$-algebras. If $\psi \circ \varphi=\varphi \circ \psi$ holds for some $\psi \in \operatorname{End}_{R}(M)$, then there exists an $m \times m$ matrix $\mathbf{P} \in \mathcal{M}(X)$ such that $\psi(\Phi(\mathbf{f}))=\Phi(\mathbf{f P})$ for all $\mathbf{f}=\left(f_{\gamma}(z)\right)_{\gamma \in \Gamma}$ in $(R[z])^{\Gamma}$. Thus $\Lambda: \mathcal{M}(X)^{\mathrm{op}} \longrightarrow \operatorname{Cen}(\varphi)$ is surjective.

2.6.Lemma. $\mathcal{I}(X) \subseteq \operatorname{ker}(\Lambda)$ ( $\Lambda$ is defined in Theorem 2.5). If $R$ is a local ring then $\mathcal{I}(X)=\operatorname{ker}(\Lambda)$.

\section{THE ZERO-LEVEL CENTRALIZER OF A NILPOTENT ENDOMORPHISM}

We keep all settings from Section 2 and define the subsets of $\mathrm{M}_{m}(R[z])$ as follows:

$$
\mathcal{M}_{0}(X)=\left\{\mathbf{P} \in \mathcal{M}(X) \mid z \mathbf{f P} \in \operatorname{ker}(\Phi) \text { for all } \mathbf{f} \in(R[z])^{\Gamma}\right\},
$$

$\mathcal{N}_{0}(X)=\left\{\mathbf{P} \in \mathrm{M}_{m}(R[z]) \mid \mathbf{P}=\left[p_{\delta, \gamma}(z)\right]\right.$ and $p_{\delta, \gamma}(z) \in J[z]+\left(z^{k_{\gamma}-1}\right)$ for all $\left.\delta, \gamma \in \Gamma\right\}$. Since $p_{\delta, \gamma}(z) \in J[z]+\left(z^{k_{\gamma}-1}\right)$ and $z p_{\delta, \gamma}(z) \in J[z]+\left(z^{k_{\gamma}}\right)$ are equivalent, we have

$$
\mathcal{N}_{0}(X)=\left[\begin{array}{cccc}
J[z]+\left(z^{k_{1}-1}\right) & J[z]+\left(z^{k_{2}-1}\right) & \cdots & J[z]+\left(z^{k_{m}-1}\right) \\
J[z]+\left(z^{k_{1}-1}\right) & J[z]+\left(z^{k_{2}-1}\right) & \cdots & J[z]+\left(z^{k_{m}-1}\right) \\
\vdots & \vdots & \ddots & \vdots \\
J[z]+\left(z^{k_{1}-1}\right) & J[z]+\left(z^{k_{2}-1}\right) & \cdots & J[z]+\left(z^{k_{m}-1}\right)
\end{array}\right] .
$$

3.1.Lemma. $\mathcal{I}(X) \subseteq \mathcal{N}_{0}(X),\left(z \mathrm{M}_{m}(R[z])\right)^{n-1} \subseteq \mathcal{N}_{0}(X), \mathcal{N}_{0}(X) \triangleleft_{l} \mathrm{M}_{m}(R[z])$ is a left ideal and $\mathcal{N}_{0}(X) \triangleleft \mathcal{N}(X)$ is an ideal. If $R$ is a local ring, then $\mathcal{N}_{0}(X)=\mathcal{M}_{0}(X)$.

Proof. The containment $\mathcal{I}(X) \subseteq \mathcal{N}_{0}(X)$ obviously holds and $\left(z \mathrm{M}_{m}(R[z])\right)^{n-1} \subseteq$ $\mathcal{N}_{0}(X)$ is a consequence of $\left(z^{n-1}\right) \subseteq\left(z^{k_{\gamma}-1}\right)$. Since the $\gamma$-th column of the matrices in $\mathcal{N}_{0}(X)$ comes from a (left) ideal $J[z]+\left(z^{k_{\gamma}-1}\right)$ of $R[z]$, we can see that $\mathcal{N}_{0}(X)$ is a left ideal of $\mathrm{M}_{m}(R[z])$. 
If $\mathbf{P} \in \mathcal{N}_{0}(X)$ and $\mathbf{Q} \in \mathcal{N}(X)$, then we have $z p_{\delta, \tau}(z) \in J[z]+\left(z^{k_{\tau}}\right)$ and $q_{\tau, \gamma}(z) \in$ $J[z]+\left(z^{k_{\tau, \gamma}}\right)$. Since $k_{\tau}+k_{\tau, \gamma} \geq k_{\gamma}$, it follows that $z p_{\delta, \tau}(z) q_{\tau, \gamma}(z) \in J[z]+\left(z^{k_{\gamma}}\right)$. Thus $\mathbf{P Q} \in \mathcal{N}_{0}(X)$ and $\mathcal{N}_{0}(X)$ is an ideal of $\mathcal{N}(X)$.

If $R$ is a local ring, then Lemma 2.3 gives that $\operatorname{ker}(\Phi)=\amalg_{\gamma \in \Gamma}\left(J[z]+\left(z^{k_{\gamma}}\right)\right)$. Let $\mathbf{1}_{\delta}$ denote the vector with 1 in its $\delta$-coordinate and zeros in all other places. If $\mathbf{P} \in \mathcal{M}_{0}(X)$, then $z \mathbf{1}_{\delta} \mathbf{P} \in \operatorname{ker}(\Phi)$ implies that $z p_{\delta, \gamma}(z) \in J[z]+\left(z^{k_{\gamma}}\right)$, whence $\mathbf{P} \in \mathcal{N}_{0}(X)$ follows. If $\mathbf{P} \in \mathcal{N}_{0}(X)$ and $\mathbf{f}=\left(f_{\gamma}(z)\right)_{\gamma \in \Gamma}$ is in $(R[z])^{\Gamma}$, then $z p_{\delta, \gamma}(z) \in$ $J[z]+\left(z^{k_{\gamma}}\right)$ implies that $z f_{\delta}(z) p_{\delta, \gamma}(z) \in J[z]+\left(z^{k_{\gamma}}\right)$ for all $\delta \in \Gamma$. Thus $z \mathbf{f P} \in \operatorname{ker}(\Phi)$ and $\mathbf{P} \in \mathcal{M}_{0}(X)$ follows.

3.2.Lemma. $\operatorname{ker}(\Lambda) \subseteq \mathcal{M}_{0}(X)$ and for $\mathbf{P} \in \mathcal{M}(X)$ the containments $\mathbf{P} \in \mathcal{M}_{0}(X)$ and $\Lambda(\mathbf{P}) \in \operatorname{Cen}_{0}(\varphi)$ are equivalent. The preimage $\mathcal{M}_{0}(X)=\Lambda^{-1}\left(\operatorname{Cen}_{0}(\varphi)\right) \triangleleft \mathcal{M}(X)$ is anideal.

Proof. The proof is based on the use of Lemma 2.3 and Theorem 2.5.

If $\mathbf{P} \in \operatorname{ker}(\Lambda)$, then $\Lambda(\mathbf{P})=\psi_{\mathbf{P}}=0$ gives that $\Phi(\mathbf{f P})=\psi_{\mathbf{P}}(\Phi(\mathbf{f}))=0$ for all $\mathbf{f} \in(R[z])^{\Gamma}$. Since $\Phi: \amalg_{\gamma \in \Gamma} R[z] \rightarrow M$ is a left $R[z]$-homomorphism, $\Phi(z \mathbf{f P})=$ $z * \Phi(\mathbf{f P})=0$ implies that $z \mathbf{f P} \in \operatorname{ker}(\Phi)$. In view of $\operatorname{ker}(\Lambda) \subseteq \mathcal{M}(X)$, we deduce that $\mathbf{P} \in \mathcal{M}_{0}(X)$.

If $\mathbf{P} \in \mathcal{M}_{0}(X)$, then $\Lambda(\mathbf{P})=\psi_{\mathbf{P}}$ and $\varphi\left(\psi_{\mathbf{P}}(\Phi(\mathbf{f}))\right)=\varphi(\Phi(\mathbf{f P}))=\Phi(z \mathbf{f P})=0$ for all $\mathbf{f} \in(R[z])^{\Gamma}$. Thus $\psi_{\mathbf{P}} \circ \varphi=\varphi \circ \psi_{\mathbf{P}}=0$ and hence $\psi_{\mathbf{P}} \in \operatorname{Cen}_{0}(\varphi)$.

If $\Lambda(\mathbf{P})=\psi_{\mathbf{P}}$ is in $\operatorname{Cen}_{0}(\varphi)$, then $\varphi \circ \psi_{\mathbf{P}}=0$ and

$$
\Phi(z \mathbf{f P})=\varphi(\Phi(\mathbf{f P}))=\varphi\left(\psi_{\mathbf{P}}(\Phi(\mathbf{f}))\right)=0
$$

for all $\mathbf{f} \in(R[z])^{\Gamma}$. It follows that $\mathbf{P} \in \mathcal{M}_{0}(X)$.

Obviously, the preimage of the ideal $\operatorname{Cen}_{0}(\varphi) \triangleleft \operatorname{Cen}(\varphi)$ is also an ideal.

3.3.Theorem. Let $\varphi \in \operatorname{End}_{R}(M)$ be a nilpotent $R$-endomorphism of a finitely generated semisimple left $R$-module ${ }_{R} M$. The map $\Lambda: \mathcal{M}(X)^{\mathrm{op}} \longrightarrow \operatorname{Cen}(\varphi)$ induces the following $Z(R)$-isomorphisms for the factor algebras:

$$
\mathcal{M}_{0}(X)^{\mathrm{op}} / \operatorname{ker}(\Lambda) \cong \operatorname{Cen}_{0}(\varphi) \text { and } \mathcal{M}(X)^{\mathrm{op}} / \mathcal{M}_{0}(X) \cong \operatorname{Cen}(\varphi) / \operatorname{Cen}_{0}(\varphi)
$$

Proof. We have $\operatorname{ker}\left(\Lambda \uparrow \mathcal{M}_{0}(X)\right)=\operatorname{ker}(\Lambda)$ and $\mathcal{M}_{0}(X)=\Lambda^{-1}\left(\operatorname{Cen}_{0}(\varphi)\right)$ by Lemma 3.2. Thus Theorem 2.5 ensures that the restricted map $\Lambda \uparrow \mathcal{M}_{0}(X)$ is a surjective $\mathcal{M}_{0}(X)^{\mathrm{op}} \longrightarrow \mathrm{Cen}_{0}(\varphi)$ homomorphism of $Z(R)$-algebras, whence $\mathcal{M}_{0}(X)^{\mathrm{op}} / \operatorname{ker}(\Lambda) \cong \operatorname{Cen}_{0}(\varphi)$ follows.

In view of Lemma 3.2, the assignment

$$
\mathbf{P}+\mathcal{M}_{0}(X) \longmapsto \Lambda(\mathbf{P})+\operatorname{Cen}_{0}(\varphi)
$$

is well-defined and gives an injective $\mathcal{M}(X)^{\mathrm{op}} / \mathcal{M}_{0}(X) \rightarrow \operatorname{Cen}(\varphi) / \operatorname{Cen}_{0}(\varphi)$ homomorphism of $Z(R)$-algebras. The surjectivity of this homomorphism is a consequence of the surjectivity of $\Lambda$ (see Theorem 2.5).

3.4.Theorem. Let $\varphi \in \operatorname{End}_{R}(M)$ be a nilpotent $R$-endomorphism of a finitely generated semisimple left $R$-module ${ }_{R} M$. If $R$ is a local ring, then the zero-level centralizer $\operatorname{Cen}_{0}(\varphi)$ of $\varphi$ is isomorphic to the opposite of the factor $\mathcal{N}_{0}(X) / \mathcal{I}(X)$ as a $Z(R)$-algebra:

$$
\operatorname{Cen}_{0}(\varphi) \cong\left(\mathcal{N}_{0}(X) / \mathcal{I}(X)\right)^{\mathrm{op}}=\mathcal{N}_{0}(X)^{\mathrm{op}} / \mathcal{I}(X)
$$

We also have an isomorphism

$$
\operatorname{Cen}(\varphi) / \operatorname{Cen}_{0}(\varphi) \cong\left(\mathcal{N}(X) / \mathcal{N}_{0}(X)\right)^{\mathrm{op}}=\mathcal{N}(X)^{\mathrm{op}} / \mathcal{N}_{0}(X)
$$


of the factor $Z(R)$-algebras.

Proof. Directly follows from Lemmas 2.4, 2.6, 3.1 and Theorem 3.3.

Define a left ideal of $\mathrm{M}_{m}(R / J)$ as follows:

$$
\mathcal{W}(X)=\left\{W=\left[w_{\delta, \gamma}\right] \mid w_{\delta, \gamma} \in R / J \text { and } w_{\delta, \gamma}=0 \text { if } k_{\gamma} \geq 2\right\} .
$$

The assumption $k_{1} \geq k_{2} \geq \cdots \geq k_{m} \geq 1$ ensures that

$$
\mathcal{W}(X)=\left[\begin{array}{cccccc}
0 & \cdots & 0 & R / J & \cdots & R / J \\
0 & \ddots & 0 & R / J & \cdots & R / J \\
\vdots & \vdots & \ddots & \vdots & \vdots & \vdots \\
\vdots & \vdots & 0 & R / J & \cdots & R / J \\
\vdots & \vdots & \vdots & \vdots & \ddots & \vdots \\
0 & \cdots & 0 & R / J & \cdots & R / J
\end{array}\right]
$$

3.5.Lemma. $\left(\mathcal{N}_{0}(X) \cap z \mathrm{M}_{m}(R[z])\right)+\mathcal{I}(X) \triangleleft \mathcal{N}_{0}(X)$ is an ideal and there is a natural ring isomorphism

$$
\mathcal{N}_{0}(X) /\left(\left(\mathcal{N}_{0}(X) \cap z \mathrm{M}_{m}(R[z])\right)+\mathcal{I}(X)\right) \cong \mathcal{W}(X)
$$

which is an $(R, R)$-bimodule isomorphism at the same time.

Proof. If $\mathbf{P}=\left[p_{\delta, \gamma}(z)\right]$ is in $\mathcal{N}_{0}(X)$ and $p_{\delta, \gamma}(z)$ has constant term $u_{\delta, \gamma} \in R$, then

$$
p_{\delta, \gamma}(z)-u_{\delta, \gamma} \in\left(J[z]+\left(z^{k_{\gamma}-1}\right)\right) \cap(z R[z])
$$

and $k_{\gamma} \geq 2$ implies that $u_{\delta, \gamma} \in J$. Thus $\left[u_{\delta, \gamma}\right] \in \mathrm{M}_{m}(R) \cap \mathcal{N}_{0}(X)$ and

$$
\mathbf{P}+\left(\left(\mathcal{N}_{0}(X) \cap z \mathrm{M}_{m}(R[z])\right)+\mathcal{I}(X)\right)=\left[u_{\delta, \gamma}\right]+\left(\left(\mathcal{N}_{0}(X) \cap z \mathrm{M}_{m}(R[z])\right)+\mathcal{I}(X)\right)
$$

holds in $\mathcal{N}_{0}(X) /\left(\left(\mathcal{N}_{0}(X) \cap z \mathrm{M}_{m}(R[z])\right)+\mathcal{I}(X)\right)$. The assignment

$$
\mathbf{P}+\left(\left(\mathcal{N}_{0}(X) \cap z \mathrm{M}_{m}(R[z])\right)+\mathcal{I}(X)\right) \longmapsto\left[u_{\delta, \gamma}+J\right]
$$

is well-defined and gives an

$$
\mathcal{N}_{0}(X) /\left(\left(\mathcal{N}_{0}(X) \cap z \mathrm{M}_{m}(R[z])\right)+\mathcal{I}(X)\right) \longrightarrow \mathcal{W}(X)
$$

isomorphism.

3.6.Theorem. Let $R$ be a local ring and $\varphi \in \operatorname{End}_{R}(M)$ be a nilpotent $R$-endomorphism of a finitely generated semisimple left $R$-module ${ }_{R} M$. If $f_{i}\left(x_{1}, \ldots, x_{r}\right) \in Z(R)\left\langle x_{1}, \ldots, x_{r}\right\rangle$, $1 \leq i \leq n$ and $f_{i}=0$ are polynomial identities of the right ideal $\mathcal{W}(X)$ of $\mathrm{M}_{m}^{\mathrm{op}}(R / J)$, then $f_{1} f_{2} \cdots f_{n}=0$ is an identity of $\operatorname{Cen}_{0}(\varphi)$.

Proof. Theorem 3.4 ensures that $\operatorname{Cen}_{0}(\varphi) \cong \mathcal{N}_{0}(X)^{\mathrm{op}} / \mathcal{I}(X)$ as $Z(R)$-algebras, hence

$$
Q=\left(\left(\mathcal{N}_{0}(X) \cap z \mathrm{M}_{m}(R[z])\right)+\mathcal{I}(X)\right) / \mathcal{I}(X) \triangleleft \mathcal{N}_{0}(X) / \mathcal{I}(X)
$$

can be viewed as an ideal of $\operatorname{Cen}_{0}(\varphi)$. The use of Lemma 3.5 gives $\operatorname{Cen}_{0}(\varphi) / Q \cong\left(\mathcal{N}_{0}(X)^{\mathrm{op}} / \mathcal{I}(X)\right) / Q \cong \mathcal{N}_{0}(X)^{\mathrm{op}} /\left(\mathcal{N}_{0}(X) \cap z \mathrm{M}_{m}(R[z])\right)+\mathcal{I}(X) \cong \mathcal{W}(X)^{\mathrm{op}}$. It follows that $f_{i}=0$ is an identity of $\operatorname{Cen}_{0}(\varphi) / Q$. Thus $f_{i}\left(v_{1}, \ldots, v_{r}\right) \in Q$ for all $v_{1}, \ldots, v_{r} \in \operatorname{Cen}_{0}(\varphi)$, and so

$$
f_{1}\left(v_{1}, \ldots, v_{r}\right) f_{2}\left(v_{1}, \ldots, v_{r}\right) \cdots f_{n}\left(v_{1}, \ldots, v_{r}\right) \in Q^{n} .
$$


Since $\left(z \mathrm{M}_{m}(R[z])\right)^{n} \subseteq \mathcal{I}(X)$ (see Lemma 2.4) implies that $Q^{n}=\{0\}$, the proof is complete.

The assumption $k_{1} \geq k_{2} \geq \ldots \geq k_{m} \geq 1$ ensures that

$$
\mathcal{U}_{0}(X)=\left\{U \in \mathrm{M}_{m}(R / J) \mid U=\left[u_{\delta, \gamma}\right] \text { and } u_{\delta, \gamma}=0 \text { if } 1 \leq k_{\delta}<k_{\gamma} \text { or } k_{\gamma}=1\right\} .
$$

is a block upper triangular subalgebra of $\mathrm{M}_{m}(R / J)$. If $\left[u_{\delta, \gamma}\right] \in \mathcal{U}_{0}(X)$ and $u_{\delta, \gamma} \neq 0$ for some $\delta, \gamma \in \Gamma$, then $2 \leq k_{\gamma} \leq k_{\delta}$. Results about the polynomial identities of block upper triangular matrix algebras can be found in [GiZ].

3.7.Lemma. There is a natural ring isomorphism

$$
\mathcal{N}(X) /\left(\left(\mathcal{N}(X) \cap z \mathrm{M}_{m}(R[z])\right)+\mathcal{N}_{0}(X)\right) \cong \mathcal{U}_{0}(X)
$$

which is an $(R, R)$-bimodule isomorphism at the same time.

Proof. For a matrix $\mathbf{P}=\left[p_{\delta, \gamma}(z)\right]$ in $\mathcal{N}(X)$ consider the assignment

$$
\mathbf{P}+\left(\left(\mathcal{N}(X) \cap z \mathrm{M}_{m}(R[z])\right)+\mathcal{N}_{0}(X)\right) \longmapsto\left[u_{\delta, \gamma}+J\right],
$$

where $u_{\delta, \gamma} \in R$ is defined as follows: $u_{\delta, \gamma}=0$ if $k_{\gamma}=1$ and $u_{\delta, \gamma}$ is the constant term of $p_{\delta, \gamma}(z)$ if $k_{\gamma} \geq 2$. Clearly, $\left[u_{\delta, \gamma}\right] \in \mathrm{M}_{m}(R) \cap \mathcal{N}(X)$ and

$\mathbf{P}+\left(\left(\mathcal{N}(X) \cap z \mathrm{M}_{m}(R[z])\right)+\mathcal{N}_{0}(X)\right)=\left[u_{\delta, \gamma}\right]+\left(\left(\mathcal{N}(X) \cap z \mathrm{M}_{m}(R[z])\right)+\mathcal{N}_{0}(X)\right)$.

In view of the definitions of $\mathcal{N}_{0}(X)$ and $\mathcal{U}_{0}(X)$, the above equality ensures that our assignment is a well-defined

$$
\mathcal{N}(X) /\left(\left(\mathcal{N}(X) \cap z \mathrm{M}_{m}(R[z])\right)+\mathcal{N}_{0}(X)\right) \longrightarrow \mathcal{U}_{0}(X)
$$

map providing the required isomomorphism.

3.8. Theorem. Let $R$ be a local ring and $\varphi \in \operatorname{End}_{R}(M)$ be a nilpotent $R$-endomorphism of a finitely generated semisimple left $R$-module ${ }_{R} M$. If $f_{i}\left(x_{1}, \ldots, x_{r}\right) \in Z(R)\left\langle x_{1}, \ldots, x_{r}\right\rangle$, $1 \leq i \leq n-1$ and $f_{i}=0$ are polynomial identities of the $Z(R)$-subalgebra $\mathcal{U}_{0}(X)$ of $\mathrm{M}_{m}^{\mathrm{op}}(R / J)$, then $f_{1} f_{2} \cdots f_{n-1}=0$ is an identity of the factor $\operatorname{Cen}(\varphi) / \operatorname{Cen}_{0}(\varphi)$.

Proof. Theorem 3.4 ensures that $\operatorname{Cen}(\varphi) / \operatorname{Cen}_{0}(\varphi) \cong \mathcal{N}(X)^{\text {op }} / \mathcal{N}_{0}(X)$ as $Z(R)$ algebras, hence

$$
L=\left(\left(\mathcal{N}(X) \cap z \mathrm{M}_{m}(R[z])\right)+\mathcal{N}_{0}(X)\right) / \mathcal{N}_{0}(X) \triangleleft \mathcal{N}(X) / \mathcal{N}_{0}(X)
$$

can be viewed as an ideal of $\operatorname{Cen}(\varphi) / \operatorname{Cen}_{0}(\varphi)$. The use of Lemma 3.7 gives

$$
\begin{gathered}
\left(\operatorname{Cen}(\varphi) / \operatorname{Cen}_{0}(\varphi)\right) / L \cong\left(\mathcal{N}(X)^{\mathrm{op}} / \mathcal{N}_{0}(X)\right) / L \cong \\
\cong \mathcal{N}(X)^{\mathrm{op}} /\left(\left(\mathcal{N}(X) \cap z \mathrm{M}_{m}(R[z])\right)+\mathcal{N}_{0}(X)\right) \cong \mathcal{U}_{0}(X)^{\mathrm{op}} .
\end{gathered}
$$

It follows that $f_{i}=0$ is an identity of $\left(\operatorname{Cen}(\varphi) / \operatorname{Cen}_{0}(\varphi)\right) / L$. Thus $f_{i}\left(v_{1}, \ldots, v_{r}\right) \in$ $L$ for all $v_{1}, \ldots, v_{r} \in \operatorname{Cen}(\varphi) / \operatorname{Cen}_{0}(\varphi)$, and so

$$
f_{1}\left(v_{1}, \ldots, v_{r}\right) f_{2}\left(v_{1}, \ldots, v_{r}\right) \cdots f_{n-1}\left(v_{1}, \ldots, v_{r}\right) \in L^{n-1} .
$$

Since $\left(z \mathrm{M}_{m}(R[z])\right)^{n-1} \subseteq \mathcal{N}_{0}(X)$ (see Lemma 3.1) implies that $L^{n-1}=\{0\}$, the proof is complete.

\section{THE ZERO-LEVEL CENTRALIZER OF AN ARBITRARY ENDOMORPHISM}

4.1.Theorem. Let $\varphi \in \operatorname{End}_{R}(M)$ be an $R$-endomorphism of a finitely generated semisimple left $R$-module ${ }_{R} M$. Then there exist $R$-submodules $W_{1}$ and $W_{2}$ of $M$ such that $W=W_{1} \oplus W_{2}$ is a direct product, $\operatorname{ker}(\varphi) \subseteq W, \varphi(W)=W_{2}, \operatorname{dim}_{R}\left(W_{1}\right)=$ 
$\operatorname{dim}_{R}(\operatorname{ker}(\varphi)),\left(\varphi\lceil W) \in \operatorname{End}_{R}(W)\right.$ is nilpotent and for the zero-level centralizer of $\varphi$ we have $\operatorname{Cen}_{0}(\varphi) \cong T$, where

$$
T=\left\{\theta \in \operatorname{End}_{R}(W) \mid \theta\left(W_{1}\right) \subseteq \operatorname{ker}(\varphi) \text { and } \theta\left(W_{2}\right)=\{0\}\right\}=\operatorname{Cen}_{0}(\varphi \mid W)
$$

is a left ideal of

$$
\operatorname{End}_{R}^{*}(W)=\left\{\alpha \in \operatorname{End}_{R}(W) \mid \alpha(\operatorname{ker}(\varphi)) \subseteq \operatorname{ker}(\varphi)\right\}
$$

and a right ideal of

$$
\operatorname{End}_{R}^{* *}(W)=\left\{\alpha \in \operatorname{End}_{R}(W) \mid \alpha\left(W_{1}+\operatorname{ker}(\varphi)\right) \subseteq W_{1}+\operatorname{ker}(\varphi) \text { and } \alpha\left(W_{2}\right) \subseteq W_{2}\right\} .
$$

Proof. The Fitting Lemma ensures the existence of an integer $t \geq 1$ such that $\operatorname{im}\left(\varphi^{t}\right) \oplus \operatorname{ker}\left(\varphi^{t}\right)=M$ is a direct sum, where the (left) $R$-submodules

$$
V=\operatorname{im}\left(\varphi^{t}\right)=\operatorname{im}\left(\varphi^{t+1}\right)=\cdots \text { and } W=\operatorname{ker}\left(\varphi^{t}\right)=\operatorname{ker}\left(\varphi^{t+1}\right)=\cdots
$$

of ${ }_{R} M$ are uniquely determined by $\varphi$. Clearly, $\varphi(V)=V$ and $\varphi(W) \subseteq W$ and the restricted map $(\varphi \mid W) \in \operatorname{End}_{R}(W)$ is nilpotent of index $q \geq 1$, where $\operatorname{ker}\left(\varphi^{q-1}\right) \neq$ $\operatorname{ker}\left(\varphi^{q}\right)=W$. Since ${ }_{R} W$ is also finitely generated and semisimple, Theorem 2.1 provides a nilpotent Jordan normal base $X=\left\{x_{\gamma, i} \mid \gamma \in \Gamma, 1 \leq i \leq k_{\gamma}\right\}$ of ${ }_{R} W$ with respect to $\varphi \uparrow W$ (we have $x_{\gamma, k_{\gamma}+1}=0$ and $q=\max \left\{k_{\gamma} \mid \gamma \in \Gamma\right\}$ ). Now $W_{1} \oplus W_{2}=W$ is a direct sum, where

$$
W_{1}=\underset{\gamma \in \Gamma}{\oplus} R x_{\gamma, 1} \text { and } W_{2}=\underset{\gamma \in \Gamma, 1 \leq i \leq k_{\gamma}}{\oplus} R x_{\gamma, i+1} .
$$

Now we have $\operatorname{ker}(\varphi) \subseteq \operatorname{ker}\left(\varphi^{t}\right)=W$ and $\operatorname{ker}(\varphi)=\operatorname{ker}(\varphi \uparrow W)=\underset{\gamma \in \Gamma}{\oplus} R x_{\gamma, k_{\gamma}}$ by Theorem 2.2. It follows that

$$
\operatorname{dim}_{R}\left(W_{1}\right)=|\Gamma|=\operatorname{dim}_{R}(\operatorname{ker}(\varphi)) .
$$

The definition of the nilpotent Jordan normal base ensures that $\varphi(W)=W_{2}$. If $\theta \in T$, then

$$
\theta(\operatorname{ker}(\varphi)) \subseteq \theta\left(W_{1} \oplus W_{2}\right)=\theta\left(W_{1}\right)+\theta\left(W_{2}\right) \subseteq \operatorname{ker}(\varphi)
$$

implies that $T$ is a left ideal of $\operatorname{End}_{R}^{*}(W)$ and a right ideal of $\operatorname{End}_{R}^{* *}(W)$. Clearly, $T=\operatorname{Cen}_{0}\left(\varphi\lceil W)\right.$ is a consequence of $\varphi(W)=W_{2}$ and the fact that $\theta(W) \subseteq \operatorname{ker}(\varphi)$ for all $\theta \in T$.

If $\alpha \in \operatorname{Cen}_{0}(\varphi)$, then $\alpha \circ \varphi=0$ implies that $\alpha(V)=\{0\}$ and $\alpha\left(x_{\gamma, i+1}\right)=$ $\alpha\left(\varphi\left(x_{\gamma, i}\right)\right)=0$ for $1 \leq i \leq k_{\gamma}-1$. We also have $\varphi \circ \alpha=0$, whence $\varphi\left(\alpha\left(x_{\gamma, 1}\right)\right)=0$ and $\alpha\left(x_{\gamma, 1}\right) \in \operatorname{ker}(\varphi)$ follow. Thus $\alpha\left(W_{2}\right)=\{0\}, \alpha\left(W_{1}\right) \subseteq \operatorname{ker}(\varphi)$ and the assignment $\alpha \longmapsto \alpha \uparrow W$ obviously defines a $\operatorname{Cen}_{0}(\varphi) \longrightarrow T$ ring homomorphism.

If $\alpha, \beta \in \operatorname{Cen}_{0}(\varphi)$ and $\alpha \uparrow W=\beta \uparrow W$, then $\alpha(V)=\beta(V)=\{0\}$ and $V \oplus W=M$ ensure that $\alpha=\beta$ proving the injectivity of the above map.

If $\theta \in T$ and $\pi_{W}: V \oplus W \longrightarrow W$ is the natural projection, then $\theta \circ \pi_{W} \in \operatorname{Cen}_{0}(\varphi)$. Indeed, $\varphi \circ \theta \circ \pi_{W}=0$ is a consequence of $\theta(W) \subseteq \operatorname{ker}(\varphi)$ and $\theta \circ \pi_{W} \circ \varphi=0$ is a consequence of $\varphi(W)=W_{2}$ and $\theta\left(W_{2}\right)=\{0\}$. Hence the surjectivity of our assignment follows from $\theta \circ \pi_{W}\lceil W=\theta$.

4.2. Corollary. Let $A \in \mathrm{M}_{n}(K)$ be an $n \times n$ matrix over a field $K$, then the $K$-dimension of the zero-level centralizer of $A$ in $\mathrm{M}_{n}(K)$ is

$$
\operatorname{dim}_{K} \operatorname{Cen}_{0}(A)=\left[\operatorname{dim}_{K}(\operatorname{ker}(A))\right]^{2} .
$$


Proof. Now $A \in \operatorname{End}_{K}\left(K^{n}\right)$ and Theorem 4.1 ensures that $\operatorname{Cen}_{0}(A) \cong T$, where

$$
T=\left\{\theta \in \operatorname{End}_{K}(W) \mid \theta\left(W_{1}\right) \subseteq \operatorname{ker}(A) \text { and } \theta\left(W_{2}\right)=\{0\}\right\} .
$$

Our claim follows from the observation that the elements of $T$ and $\operatorname{Hom}_{K}\left(W_{1}, \operatorname{ker}(A)\right)$ can be naturally identified and $\operatorname{dim}_{K}\left(W_{1}\right)=\operatorname{dim}_{K}(\operatorname{ker}(A))$.

Remark. Theorem 4.1 shows that the determination of the zero-level centralizer can be reduced to the nilpotent case. This reduction depends on the use of the Fitting Lemma.

4.3.Lemma. Let $\varphi, \sigma \in \operatorname{End}_{R}(M)$ be R-endomorphisms of a finitely generated semisimple left $R$-module ${ }_{R} M$. If $\operatorname{Cen}_{0}(\varphi) \subseteq \operatorname{Cen}_{0}(\sigma)$, then $\operatorname{ker}(\varphi) \subseteq \operatorname{ker}(\sigma)$.

Proof. We use the proof of Theorem 4.1. If $\gamma \in \Gamma$ and $\pi_{\gamma} \in \operatorname{End}_{R}(M)$ denotes the natural

$$
M=V \oplus W=V \oplus\left(\underset{\delta \in \Gamma, 1 \leq i \leq k_{\delta}}{\oplus} R x_{\delta, i}\right) \longrightarrow R x_{\gamma, k_{\gamma}}
$$

projection, then $\pi_{\gamma} \circ \varphi^{k_{\gamma}-1} \in \operatorname{Cen}_{0}(\varphi)$. It follows that $\pi_{\gamma} \circ \varphi^{k_{\gamma}-1} \in \operatorname{Cen}_{0}(\sigma)$, whence we obtain that $\sigma \circ \pi_{\gamma} \circ \varphi^{k_{\gamma}-1}=0$. Since $\sigma\left(x_{\gamma, k_{\gamma}}\right)=\sigma\left(\pi_{\gamma}\left(\varphi^{k_{\gamma}-1}\left(x_{\gamma, k_{1}}\right)\right)\right)=0$, we have $x_{\gamma, k_{\gamma}} \in \operatorname{ker}(\sigma)$ for all $\gamma \in \Gamma$. Thus

$$
\operatorname{ker}(\varphi)=\operatorname{ker}\left(\varphi\lceil W)=\underset{\gamma \in \Gamma}{\oplus} R x_{\gamma, k_{\gamma}} \subseteq \operatorname{ker}(\sigma) .\right.
$$

For a matrix $A \in \mathrm{M}_{n}(K)$ let $A^{\top}$ denote the transpose of $A$.

4.4.Theorem. If $A, B \in \mathrm{M}_{n}(K)$ are $n \times n$ matrices over a field $K$, then the following are equivalent:

1. $\operatorname{Cen}_{0}(A) \subseteq \operatorname{Cen}_{0}(B)$

2. $\operatorname{ker}(A) \subseteq \operatorname{ker}(B)$ and $\operatorname{ker}\left(A^{\top}\right) \subseteq \operatorname{ker}\left(B^{\top}\right)$.

Proof. (1) $\Longrightarrow(2)$ : For a matrix $C \in \operatorname{Cen}_{0}\left(A^{\top}\right)$ we have $C A^{\top}=A^{\top} C=0$ and $C^{\top} \in \operatorname{Cen}_{0}(A)$ is a consequence of

$$
A C^{\top}=\left(A^{\top}\right)^{\top} C^{\top}=\left(C A^{\top}\right)^{\top}=0=\left(A^{\top} C\right)^{\top}=C^{\top}\left(A^{\top}\right)^{\top}=C^{\top} A .
$$

Thus $C^{\top} \in \operatorname{Cen}_{0}(B)$ and a similar argument gives that $C=\left(C^{\top}\right)^{\top} \in \operatorname{Cen}_{0}\left(B^{\top}\right)$. It follows that $\operatorname{Cen}_{0}\left(A^{\top}\right) \subseteq \operatorname{Cen}_{0}\left(B^{\top}\right)$. The application of Lemma 4.3 for the matrices $A, B, A^{\top}, B^{\top} \in \operatorname{End}_{K}\left(K^{n}\right)$ gives $\operatorname{ker}(A) \subseteq \operatorname{ker}(B)$ and $\operatorname{ker}\left(A^{\top}\right) \subseteq \operatorname{ker}\left(B^{\top}\right)$.

$(2) \Longrightarrow(1)$ : For a matrix $C \in \operatorname{Cen}_{0}(A)$ the containment $\operatorname{im}(C) \subseteq \operatorname{ker}(A)$ is a consequence of $A C=0$ and $\operatorname{im}\left(C^{\top}\right) \subseteq \operatorname{ker}\left(A^{\top}\right)$ is a consequence of $A^{\top} C^{\top}=$ $(C A)^{\top}=0$. Now $\operatorname{im}(C) \subseteq \operatorname{ker}(B)$ implies that $B C=0$ and $\operatorname{im}\left(C^{\top}\right) \subseteq \operatorname{ker}\left(B^{\top}\right)$ implies that $C B=\left(B^{\top} C^{\top}\right)^{\top}=0$. Thus $C \in \operatorname{Cen}_{0}(B)$ and $\operatorname{Cen}_{0}(A) \subseteq \operatorname{Cen}_{0}(B)$ follows.

ACKNOWLEDGMENT: The authors wish to thank P.N. Anh and L. Marki for fruitful consultations.

\section{REFERENCES}

[DSzW] Drensky, V., Szigeti, J. and van Wyk, L. Centralizers in endomorphism rings, J. Algebra 324 (2010), 3378-3387. 
[Ga] Gantmacher, F.R. The Theory of Matrices, Chelsea Publishing Co., New York, 2000.

[GiZ] Giambruno, A. and Zaicev, M. Polynomial Identities and Asymptotic Methods, Mathematical Surveys and Monographs 122, Amer. Math. Soc., Providence, Rhode Island, 2005.

[P] Prasolov, V.V. Problems and Theorems in Linear Algebra, Vol. 134 of Translation of Mathematical Monographs, Amer. Math. Soc., Providence, Rhode Island, 1994.

[SuTy] Suprunenko, D.A. and Tyshkevich, R.I. Commutative Matrices, Academic Press, New York and London, 1968.

[Sz] Szigeti, J. Linear algebra in lattices and nilpotent endomorphisms of semisimple modules, J. Algebra 319 (2008), 296-308.

[TuA] Turnbull, H.W. and Aitken, A.C. An Introduction to the Theory of Canonical Matrices, Dover Publications, 2004.

Institute of Mathematics, University of Miskold, Miskold, Hungary 3515

E-mail address: jeno.szigeti@uni-miskolc.hu

Department of Mathematical Sciences, Stellenbosch University, P/Bag X1, Matieland 7602, Stellenbosch, South Africa

E-mail address: LvW@sun.ac.za 